\title{
TANGENTS TO SUBSOLUTIONS EXISTENCE AND UNIQUENESS, II
}

F. Reese Harvey and H. Blaine Lawson, Jr.*

\begin{abstract}
This part II of the paper is concerned with questions of existence and uniqueness of tangents in the special case of $\mathbb{G}$-plurisubharmonic functions, where $\mathbb{G} \subset G\left(p, \mathbf{R}^{n}\right)$ is a compact subset of the Grassmannian of $p$-planes in $\mathbf{R}^{n}$. An u.s.c. function $u$ on an open set $\Omega \subset \mathbf{R}^{n}$ is $\mathbf{G}$-plurisubharmonic if its restriction to $\Omega \cap W$ is subharmonic for every affine $\mathbb{G}$-plane $W$. Here $\mathbb{G}$ is assumed to be invariant under a subgroup $K \subset \mathrm{O}(n)$ which acts transitively on $S^{n-1}$. Tangents to $u$ at a point $x$ are the cluster points of $u$ under a natural flow (or blow-up) at $x$. They always exist and are $\mathbb{G}$-harmonic at all points of continuity. A homogeneity property is established for all tangents in these geometric cases. This leads to principal results concerning the Strong Uniqueness of Tangents, which means that all tangents are unique and of the form $\Theta K_{p}$ where $K_{p}$ is the Riesz kernel and $\Theta$ is the density of $u$ at the point. Strong uniqueness is a form of regularity which implies that the sets $\{\Theta(u, x) \geq c\}$ for $c>0$ are discrete. When the invariance group $K=\mathrm{O}(n), \mathrm{U}(n)$ or $\operatorname{Sp}(n)$ strong uniqueness holds for all but a small handful of cases. It also holds for essentially all interesting $\mathbb{G}$ which arise in calibrated geometry.

When strong uniqueness fails, homogeneity implies that tangents are characterized by a subequation on the sphere, which is worked out in detail. In the cases corresponding to the real, complex and quaternionic Monge-Ampère equations (convex functions, and complex and quaternionic plurisubharmonic functions) tangents, which are far from unique, are then systematically studied and classified.
\end{abstract}

\section{TABLE OF CONTENTS}

1. Introduction.

2. The Homogeneity Theorem.

3. The Strong Uniqueness Theorem.

4. Homogeneous F-Subharmonic Functions.

5. Tangents to Convex, C-Plurisubharmonic, and $\mathbf{H}$-Plurisubharmonic Functions.

Appendix A. Further Discussion of Examples.

*Partially supported by the N.S.F. 


\section{Introduction.}

Part I of this paper was concerned with the study of tangents to $F$-subharmonic functions (or subsolutions) for any fully nonlinear subequation $F \subset \operatorname{Sym}^{2}\left(\mathbf{R}^{n}\right)$. Key to the results is the notion of the Riesz characteristic of $F$, a real number $p=p_{F}$ with $1 \leq p \leq \infty$. When $p$ is finite, there is an associated tangential $p$-flow on $F$-subharmonic functions $u$ at 0 given by

$$
u_{r}(x)=\left\{\begin{array}{cl}
r^{p-2} u(r x) & \text { if } p \neq 2, \\
u(r x)-M(u, r) & \text { if } p=2,
\end{array}\right. \text { and }
$$

where

$$
M(r) \equiv \sup _{|x| \leq r} u .
$$

Tangents to $u$ at 0 are then defined to be the cluster points of this flow in $L_{\text {loc }}^{1}\left(\mathbf{R}^{n}\right)$. A basic result is that tangents always exist, and the set of tangents to $u$ at 0 has a list of characterizing properties (Part I, Section 8). Tangents are also always maximal (Part I, Section 6). In particular, they are $F$-harmonic outside possible poles.

Of basic importance to this study is the $p^{\text {th }}$ Riesz kernel $K_{p}(|x|)$ where

$$
K_{p}(t)= \begin{cases}t^{2-p} & \text { if } 1 \leq p<2 \\ \log t & \text { if } p=2 \\ -\frac{1}{t^{p-2}} & \text { if } 2<p<\infty .\end{cases}
$$

When the Riesz characteristic $p=p_{F}$ is finite, every increasing radial $F$-harmonic is of the form $\Theta K_{p}(|x|)+C$. A fundamental Monotonicity Theorem (Part I, Section 5) states that

$$
\frac{M(u, r)-M(u, s)}{K_{p}(r)-K_{p}(s)} \text { is increasing in } r \text { and } s .
$$

for all $0<r<s$ where $M$ is defined. This gives the notion of the density of $u$ at 0 :

$$
\Theta(u, 0)=\lim _{r<s \downarrow 0} \frac{M(u, r)-M(u, s)}{K_{p}(r)-K_{p}(s)} .
$$

(When $F$ is convex, there are other densities defined via the area and volume averages.)

This part of the paper is exclusively concerned with geometric subequations determined by a closed subset $\mathbb{G} \subset G\left(p, \mathbf{R}^{n}\right)$ of the Grassmannian of unoriented $p$-planes in $\mathbf{R}^{n}$ (where $1 \leq p \leq n$ ). We recall that the associated subequation is

$$
F(\mathbb{G}) \equiv\left\{A \in \operatorname{Sym}^{2}\left(\mathbf{R}^{n}\right): \operatorname{tr}\left(\left.A\right|_{W}\right) \geq 0 \forall W \in \mathbb{G}\right\} .
$$

The Riesz characteristic of $F(\mathbb{G})$ is the integer $p$. The $F(\mathbb{G})$-subharmonic functions are called $\mathbb{G}$-plurisubharmonic, and they are characterized by the property that their restrictions to affine $\mathbb{G}$-planes are subharmonic $\left[\mathrm{HL}_{6}\right]$. Many examples of geometric interest 
are given in Part I. These include in particular the plurisubharmonics associated to any calibration, and the Lagrangian subharmonics in $\mathbf{C}^{n}$.

Recall that the standing assumptions on $F \subset \operatorname{Sym}^{2}\left(\mathbf{R}^{n}\right)$ in Part I were:

(i) (Positivity) $F+\mathcal{P} \subset F$ where $\mathcal{P}=\{A \geq 0\}$,

(ii) (Cone property) $t F=F$ for all $t \geq 0$,

(iii) (ST-Invariance) $F$ is invariant under a subgroup $G \subset \mathrm{O}(n)$ which acts transitively on the sphere $S^{n-1}$.

The first two assumptions are automatic for $F(\mathbb{G})$. The last is equivalent to the assumption that $\mathbb{G}$ is invariant under the subgroup $G \subset \mathrm{O}(n)$ acting on the Grassmannian $G\left(p, \mathbf{R}^{n}\right)$.

To state the main results we recall the following.

Definition 1.1. We say that uniqueness of tangents holds for the subequation $F$ if for every $F$-subharmonic function $u$ defined in a neighborhood of 0 , there is exactly one tangent to $u$ at 0 . We say that strong uniqueness of tangents holds for $F$ if for every such $u$, the unique tangent is $\Theta(u, 0) K_{p}(|x|)$.

Definition 1.2. An upper semi-continuous function $U: \mathbf{R}^{n} \rightarrow[-\infty, \infty)$ is said to have Riesz homogeneity $p$ if $U_{r}=U$ for all $r>0$. This condition holds if and only if there exists an u.s.c. function $g$ on the unit sphere $S$ such that

$$
U(x)=|x|^{p-2} g\left(\frac{x}{|x|}\right) \quad \text { in the cases where } p \neq 2
$$

while in the case where $p=2$,

$$
U(x)=\Theta \log |x|+g\left(\frac{x}{|x|}\right) \quad \text { with } \sup _{S^{n-1}} g=0 \quad \text { and } \quad \Theta \geq 0 \text { a constant. }
$$

Note 1.3. When $p=1$ our assumption of ST-invariance implies that $\mathbb{G}=G\left(1, \mathbf{R}^{n}\right)$. Hence, there is only one geometric subequation, namely $\mathcal{P}=\{A \geq 0\}$. The $\mathcal{P}$-subharmonic functions are exactly the convex functions, and in this case straightforward classical arguments establish the existence, uniqueness and homogeneity of tangents at every point. These proofs are omitted. On the other hand, strong uniqueness of tangents fails in this case, and the classification is given in section 5 .

Our first main result is the following. Let $\mathbb{G} \subset G\left(p, \mathbf{R}^{n}\right)$ be as above, with $p \geq 2$.

THE HOMOGENEITY THEOREM. Suppose $u$ is a $\mathbb{G}$-plurisubharmonic function defined in a neighborhood of 0 , and suppose $U$ is a tangent to $u$ at 0 . Then $U$ has Riesz homogeneity $p$. Moreover, for all $\mathbb{G}$-planes $W$ passing through the origin, the function $g$ is constant on the unit sphere $W \cap S^{n-1}$ in $W$. In fact, when $p \neq 2$,

$$
g\left(\frac{x}{|x|}\right)=-\Theta(W) \quad \text { if } \quad x \in W \in \mathbb{G}
$$

The fact that $g$ is constant on each intersection $W \cap S^{n-1}$ for $W \in \mathbb{G}$ leads to the following. We say $\mathbb{G}$ has the transitivity property if for any two vectors $x, y \in \mathbf{R}^{n}$ there exist $W_{1}, \ldots, W_{k} \in \mathbb{G}$ with $x \in W_{1}, y \in W_{k}$ and $\operatorname{dim}\left(W_{i} \cap W_{i+1}\right)>0$ for all $i=1, \ldots, k-1$. 
THE PRELIMINARY STRONG UNIQUENESS THEOREM. If $\mathbb{G}$ has the transitivity property, then strong uniqueness of tangents holds for all $\mathbb{G}$-plurisubharmonic functions.

This covers a number of interesting cases which are not included in the Strong Uniqueness Theorem of Part I. For example, this establishes strong uniqueness for plurisubharmonic functions in Special Lagrangian, associative, coassociative and Cayley geometry (See Section 3.) The invariance groups in these cases are $\mathrm{SU}(n), \mathrm{G}_{2}$ and $\operatorname{Spin}_{7}$. For the standard families of groups acting transitively on spheres we have the following nearly complete result.

THE PRINCIPAL STRONG UNIQUENESS THEOREM. Fix $p \geq 2$ and $n \geq 3$. Then strong uniqueness of tangents to $\mathbf{G}$-plurisubharmonic functions holds for:

(a) Every compact $S U(n)$-invariant subset $\mathbb{G} \subset G^{\mathbf{R}}\left(p, \mathbf{C}^{n}\right)$ except $\mathcal{P}^{\mathbf{C}}$,

(b) Every compact $\operatorname{Sp}(n) \cdot \operatorname{Sp}(1)$-invariant subset $\mathbb{G} \subset G^{\mathbf{R}}\left(p, \mathbf{H}^{n}\right)$ with three exceptions, namely the sets of real $p$-planes which lie in a quaternion line for $p=2,3,4$ (when $p=4$ this is $\mathcal{P}^{\mathbf{H}}$ ),

(c) Every compact $S p(n)$-invariant subset $\mathbb{G} \subset G^{\mathbf{R}}\left(p, \mathbf{H}^{n}\right)$, for $p \geq 5$.

We recall that by Theorem 11.1 from Part I: If strong uniqueness holds, then for every G-plurisubharmonic function $u$, the set

$$
E_{c}=\{x: \Theta(u, x) \geq c\} \text { is discrete for all } c>0 \text {. }
$$

In those cases where strong uniqueness fails we have the following question : What is the subequation on the sphere $S^{n-1}$ satisfied by the function $g$ in (1.7)? This subequation is worked out in Section 4. Its viscosity subsolutions are exactly the functions $g$ in the Homogeneity Theorem above.

The three classical cases where strong uniqueness of tangents fails are:

$$
\mathbb{G}=G^{\mathbf{R}}\left(1, \mathbf{R}^{n}\right), \quad G^{\mathbf{C}}\left(1, \mathbf{C}^{n}\right) \text { and } G^{\mathbf{H}}\left(1, \mathbf{H}^{n}\right) .
$$

The associated subequations

$$
F(\mathbb{G})=\mathcal{P}^{\mathbf{R}}, \quad \mathcal{P}^{\mathbf{C}} \text { and } \mathcal{P}^{\mathbf{H}}
$$

the homogeneous real, complex and quaternionic Monge-Ampère equations respectively. These cases are discussed in detail in Section 5.

For the first case, $\mathcal{P}^{\mathbf{R}}$-subharmonic functions are just classical convex functions. Here tangents are unique, but strong uniqueness is far from true. The results here are classical, but we review them for the light they shed on the general picture.

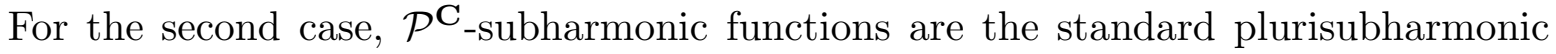
functions in $\mathbf{C}^{n}$. Here even the uniqueness of tangents fails. However, the subsets of functions in $L_{\text {loc }}^{1}\left(\mathbf{C}^{n}\right)$ which can arise as the set of tangents at 0 to a p.s.h. function $u$ have been completely classified by Kiselman $[\mathrm{K}]$ whose work was the inspiration for this paper. One new feature of our presentation is that in this case, we show that tangents correspond bijectively to quasi-plurisubharmonic functions on complex projective space $\mathbf{P}^{n-1}(\mathbf{C})$. 
For the third case, $\mathcal{P}^{\mathbf{H}}$-subharmonic functions are quaternionic plurisubharmonic functions (cf. $\left.\left[\mathrm{A}_{1,2}\right],[\mathrm{AV}]\right)$. Here the determination of tangents is new. As above, the tangents corresponds bijectively to upper semi-continuous functions $g$ on quaternionic projective space $\mathbf{P}^{n-1}(\mathbf{H})$ which satisfy the inequality $\operatorname{Hess}_{\mathbf{H}}(g)-2 g I \geq 0$ in the viscosity sense.

Finally, in Appendix A we give a rounded discussion of the many examples to which the results of both Parts I and II apply. This includes the establishment of the maximal and minimal subequations of Riesz characteristic $p$ as well as the maximal and minimal ones in the convex case.

\section{The Homogeneity Theorem.}

In this section we establish the homogeneity of tangents for all geometrically determined subequations. We assume, to begin, that $\mathbb{G} \subset G\left(p, \mathbf{R}^{n}\right)$ is a smooth compact submanifold of the Grassmannian where the integer $p$ equals $2,3, \ldots, n-1$. Later we will be able to drop this smoothness assumption and allow $\mathbb{G}$ to be any closed subset of $p$ planes. We always assume that $\mathbb{G}$ is invariant under the natural action on $G\left(p, \mathbf{R}^{n}\right)$ of a subgroup $G \subset \mathrm{O}(n)$ which acts transitively on the unit sphere $S^{n-1} \subset \mathbf{R}^{n}$. This implies in particular that every vector in $\mathbf{R}^{n}$ lies in some $\mathbb{G}$-plane

Recall that the associated subequation $F$ is given by

$$
F=F(\mathbb{G}) \equiv\left\{A \in \operatorname{Sym}^{2}\left(\mathbf{R}^{n}\right): \operatorname{tr}_{W} A \geq 0 \text { for all } W \in \mathbb{G}\right\} .
$$

The Riesz characteristic of $F$ is easily seen to be the integer $p$, and in fact, this is the reason for the choice of normalization for the Riesz kernel $K_{p}$ in (1.3).

Suppose $u$ is an $F$-subharmonic function which is defined in a neighborhood of the origin, and $U$ is a tangent to $u$ at 0 . We assume $u \not \equiv-\infty$. By the Restriction Theorem proved in $\left[\mathrm{HL}_{6}\right]$

$$
\left.u\right|_{W} \text { is Laplacian subharmonic on } W(\text { near } 0) \text { for each } W \in \mathbb{G} \text {. }
$$

In particular, either $\left.u\right|_{W}$ is $L_{\text {loc }}^{1}$ or $\left.u\right|_{W} \equiv-\infty$. We say that $W$ is non-polar for $u$ at 0 in the first case, and polar in the second case. The invariance of $\mathbb{G}$ implies that $F \subset \Delta$ (see (5.3) in Part I), and hence $u$ is $\Delta$-subharmonic near $0 \in \mathbf{R}^{n}$. Therefore, its $-\infty$ set has $\Delta$-capacity zero, and hence measure zero. This proves

The union of all polar planes $W \in \mathbb{G}$ is a set of measure zero in $\mathbf{R}^{n}$

If $W$ is non-polar for $u$ near 0 , then because of (2.1) we can apply the classical fact for the Laplacian that

$$
\left.u\right|_{W} \text { has the unique tangent function } \Theta(W) K_{p}
$$

where $K_{p}$ denote the function $K_{p}(|x|)$ and where the constant

$$
\Theta(W)=\Theta^{M}\left(\left.u\right|_{W}\right)=\Theta^{S}\left(\left.u\right|_{W}\right)
$$


is the maximum and/or spherical density of $\left.u\right|_{W}$ at 0 (see (9.3) and Proposition 9.4 in Part I). That is,

$$
\lim _{r \rightarrow 0}\left(\left.u\right|_{W}\right)_{r}=\Theta(W) K_{p} \quad \text { in } L_{\mathrm{loc}}^{1}(W)
$$

Note that these limits are over all $r$, not just a sequence $r_{j}$. Also note that for $p \geq 3$ we have

$$
\left(\left.u\right|_{W}\right)_{r}=\left.u_{r}\right|_{W} .
$$

(This does not hold for $p=2$.)

The main result of this section is the following. Recall we assume $u \not \equiv-\infty$. Extend the definition of $\Theta(W)$ to all $W \in \mathbb{G}$ by defining $\Theta(W)=+\infty$ if $W$ is polar for $u$ at 0 .

THEOREM 2.1. Suppose $u$ is an F-subharmonic function which is defined in a neighborhood of the origin, and $U$ is a tangent to $u$ at 0 . Then $U$ has Riesz homogeneity $p$, that is,

$$
U(x)=\left\{\begin{array}{cl}
\frac{1}{|x|^{p-2}} g\left(\frac{x}{|x|}\right) & \text { if } p>2 \\
\Theta \log |x|+g\left(\frac{x}{|x|}\right) & \text { if } p=2
\end{array} \quad \text { where }\left.g \equiv U\right|_{S^{n-1}} \in \operatorname{USC}\left(S^{n-1}\right) .\right.
$$

and where, in the case $p=2$, $\sup g=0$ and $\Theta=\Theta^{M}(u, 0)$. Moreover, for each $\mathbb{G}$-plane $W$ passing through the origin, the function $g$ is constant on the unit sphere $W \cap S^{n-1}$ in $W$. In fact, when $p>2$,

$$
g\left(\frac{x}{|x|}\right)=-\Theta(W) \quad \text { for } \quad x \in W \in \mathbb{G} .
$$

Proof. We first treat the case $p \geq 3$. Fix constants $0<a<b$, and let $\mathbf{A}=\left\{x \in \mathbf{R}^{n}\right.$ : $a \leq|x| \leq b\}$ be the annulus with radii $a, b$. It will suffice to prove our assertions on $\mathbf{A}$.

To begin set $G r \equiv G\left(p, \mathbf{R}^{n}\right)$ and consider the tautological vector bundle

$$
E \equiv\left\{(W, x) \in G r \times \mathbf{R}^{n}: x \in W\right\} \stackrel{\sigma}{\longrightarrow} G r
$$

where $\sigma$ is given by projection onto the first factor in $G r \times \mathbf{R}^{n}$. Projection onto the second factor gives another map

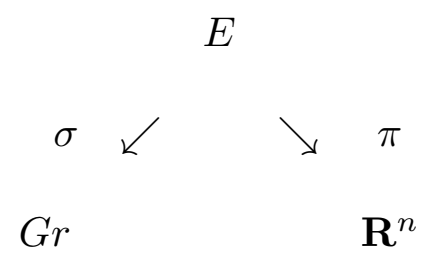

Note that $\pi: E-Z \rightarrow \mathbf{R}^{n}-\{0\}$ is a proper submersion, where $Z=\pi^{-1}(0) \subset Y$ is the zero section of the vector bundle $\sigma$. Setting $E_{\mathbf{A}}=\pi^{-1}(\mathbf{A})$ we have a pair of smooth compact fibre bundles

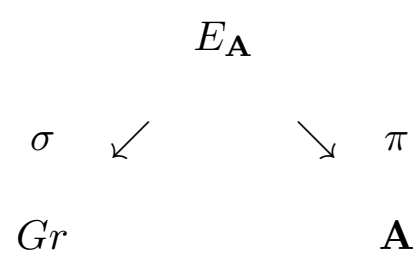


where the fibre of $\sigma$ over $W \in G r$ is the $(a, b)$-annulus in $W$. Note that the orthogonal group acts naturally on this diagram.

We now restrict this annulus-bundle $E_{\mathbf{A}}$ to the submanifold $\mathbb{G} \subset G r$, that is, we set $\mathbf{E} \equiv \sigma^{-1}(\mathbb{G})$. The diagram above reduces to a new diagram

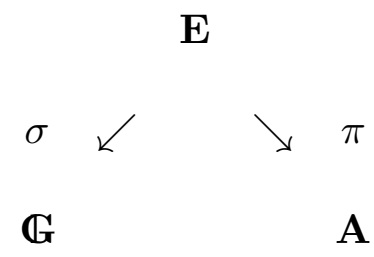

Note that the subgroup $G \subset \mathrm{O}(n)$ acts naturally on this diagram (2.7), and recall that $G$ acts transitively on the concentric spheres $S_{r}^{n-1}=\{|x|=r\}, a \leq r \leq b$, in the annular region $\mathbf{A}$. This, together with the fact that $\pi$ is a linear embedding on the fibres of $\sigma$, shows that $\mathbf{E} \stackrel{\pi}{\longrightarrow} \mathbf{A}$ is also a smooth fibre-bundle over the manifold-with-boundary $\mathbf{A}$.

We fix a defining sequence $u^{j} \equiv u_{r_{j}}$ for $U$, and consider the pull-backs $\widetilde{u}^{j} \equiv \pi^{*} u^{j}$ and $\widetilde{U} \equiv \pi^{*} U$ to $\mathbf{E}$. Note that $\widetilde{U}$ is u.s.c., in fact it is essentially u.s.c. (since $U$ is), and we have that

$$
\widetilde{u}^{j} \rightarrow \widetilde{U} \quad \text { in } L^{1}(\mathbf{E})
$$

In addition, set $\widetilde{V}(W, x) \equiv \Theta(W) K_{p}(|x|)$ if $x \neq 0$ and $(W, x) \in \mathbf{E}$, i.e., $x \in W$. Then $(2.3)^{\prime}$ implies that

$$
\left.\left.\left.\widetilde{u}^{j}\right|_{\sigma^{-1}(W)}(x) \rightarrow \widetilde{V}(W, x) \quad \text { in } L^{1}\left(\sigma^{-1}(W)\right)\right) \cong L^{1}(W \cap \mathbf{A})\right) \quad \forall W \in \mathbb{G} \text { nonpolar. }
$$

Lemma 2.2. $\widetilde{U}=\widetilde{V}$ almost everywhere on E. Furthermore,

$$
\left.U\right|_{W}=\Theta(W) K_{p} \quad \text { for almost all } W \in \mathbb{G} .
$$

Corollary 2.3. $U_{r}=U$ on $\mathbf{R}^{n}$ for all $r>0$, i.e., $U$ is $p$-homogeneous.

Proof of Corollary 2.3. By (2.10) and (2.2) we see that $U=U_{r}$ a.e. in $\mathbf{R}^{n}$. Note, however, that $U=U_{r}$ a.e. implies that $U=U_{r}$ everywhere since both functions are

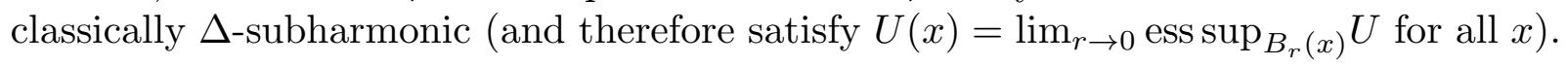

Proof of Lemma 2.2. The fibration $\sigma: \mathbf{E} \rightarrow \mathbb{G}$ is locally a product $B \times A$ where $B$ is an open ball in the manifold $\mathbb{G}$ and $A$ is the $[a, b]$-annulus in $\mathbf{R}^{p}$. Furthermore, the riemannian measure on $B \times A$ (for the metric induced from $G r \times \mathbf{R}^{n}$ ) is smoothly equivalent to the product measure. Hence, it suffices to consider the cartesian case. For simplicity we drop the tildes and rewrite the condition (2.8) as

$$
u^{j}(w, x) \rightarrow U(w, x) \quad \text { in } L^{1}(B \times A) .
$$

and rewrite the condition $(2.9)$ as

$$
u^{j}(w, x) \rightarrow V(w, x) \quad \text { in } L^{1}(A) \quad \text { for all nonpolar } w \in B
$$


By $(2.8)^{\prime}$ we have that

$$
\left|u^{j}(w, x)-U(w, x)\right| \rightarrow 0 \quad \text { in } L^{1}(B \times A)
$$

and by $(2.9)^{\prime}$ we see that for all non-polar $w \in B$,

$$
\left|u^{j}(w, x)-U(w, x)\right| \rightarrow|V(w, x)-U(w, x)| \quad \text { in } \quad L^{1}(A) .
$$

Now by the Fubini Theorem, the function

$$
I^{j}(w) \equiv \int_{A}\left|u^{j}(w, x)-U(w, x)\right| d x
$$

in integrable on $B$, and

$$
\int_{B} I^{j}(w) d w=\int_{B \times A}\left|u^{j}(w, x)-U(w, x)\right| d w d x .
$$

Moreover, by $(2.9)^{\prime \prime}$ we know that

$$
I^{j}(w) \text { converges pointwise to } \int_{A}|V(w, x)-U(w, x)| d x \text { on } B .
$$

By Fatou's Lemma and (2.12), (2.11) and (2.8)" we have

$$
\int_{B \times A}|V(w, x)-U(w, x)| d w d x=\int_{B} \lim I^{j}(w) d w \leq \underline{\lim } \int_{b} I^{j}(w) d w=0 .
$$

Thus $V=U$ a.e. on $B \times A$. Furthermore, for almost all $w \in B$, the restrictions satisfy $\left.V\right|_{w \times A}=\left.U\right|_{w \times A}$ a.e. on $A$. Since these restrictions are both $\Delta$-subharmonic on $A$, we conclude equality everywhere on $A$. This establishes (2.10) and completes the proof of Lemma 2.2 .

Finally we prove (2.6). Let $\mathbb{G}^{*} \subset \mathbb{G}$ denote the set of non-polar $\mathbb{G}$-planes $W$ for which

$$
\left.U\right|_{W}=\Theta(W) K_{p}
$$

This set has full measure in $\mathbb{G}$ by $(2.10)$.

Consider a general non-polar $p$-plane $W \in \mathbb{G}$. Let $S_{W}=W \cap S^{n-1}$ denote the unit sphere in $W$. Since $g \equiv U \mid S^{n-1}$ is upper semi-continuous on $S_{W}$, it assumes its maximum at a point $x \in S_{W}$. Now since

$$
\widetilde{U}(W, x)=\underset{\left(W^{\prime}, x^{\prime}\right) \rightarrow(W, x)}{\operatorname{ess} \limsup } \widetilde{U}\left(W^{\prime}, x^{\prime}\right)
$$

and $\mathbb{G}^{*}$ has full measure,

There exists a sequence $\left(W_{j}, x_{j}\right) \in Y$, with $\left|x_{j}\right|=1$ and $W_{j} \in \mathbb{G}^{*}$ 


$$
\text { such that }\left(W_{j}, x_{j}\right) \rightarrow(W, x) \text { and } \widetilde{U}\left(W_{j}, x_{j}\right) \rightarrow \widetilde{U}(W, x)
$$

Choose another unit vector $y \in S_{W}$. Since $W_{j} \rightarrow W$, we have $S_{W_{j}} \rightarrow S_{W}$ and there exists a sequence of unit vectors $y_{j} \in W_{j}$ such that $y_{j} \rightarrow y$. Since $W_{j} \in \mathbb{G}^{*}$, we have $g\left(x_{j}\right)=g\left(y_{j}\right)=-\Theta\left(\left.u\right|_{W_{j}}\right)$. Thus, using the upper semi-continuity of $g$ we have

$$
g(x)=\lim _{j} g\left(x_{j}\right)=\lim _{j} g\left(y_{j}\right) \leq g(y),
$$

and $g(y) \leq g(x)$ since $g(x)$ is the maximum value of $g$ on $S_{W}$. We have proved that $g$ is constant on $S_{W}$. By Corollary 2.3 and the definition of density, we now conclude (2.13) for our general non-polar plane $W$. Finally note that $\left.U\right|_{W} \equiv-\infty$ if $W$ is polar for $u$ at 0 .

We have now proved Theorem 2.1 for $p \geq 3$ under the assumption that $\mathbb{G}$ is a smooth submanifold. For a general $\mathbb{G}$, choose any point $W \in \mathbb{G}$ and consider the $G$-orbit $\mathbb{G}_{0} \equiv G \cdot W \subset \mathbb{G}$. Now $\mathbb{G}_{0}$ is a compact smooth submanifold of $G\left(p, \mathbf{R}^{n}\right)$, and since $F \subset F_{0} \equiv F\left(\mathbb{G}_{0}\right)$, we see that any $F$-subharmonic function is $F_{0}$-subharmonic. Hence the result for smooth $\mathbb{G}$ implies the result in general.

We now address the case $p=2$. The proof given here follows that of Kiselman $[\mathrm{K}]$ and is easier than the one given above for the cases $p \geq 3$. Our first observation is that by the first equality in (7.8) of Part I, we have

$$
U(x) \leq \Theta^{M}(u) \log |x| \quad x \in \mathbf{R}^{n} .
$$

Suppose now that $W \in \mathbb{G}$ is non-polar for $u$ at 0 . Using either of the complex structures induced on the 2-plane $W$ by the inner product, we have that if $x \in W$ and $\lambda \in \mathbf{C}$, then $U(\lambda x) \leq \Theta^{M}(u) \log |\lambda x|$. Hence,

$$
U(\lambda x)-\Theta^{M}(u) \log |\lambda| \leq \Theta^{M}(u) \log |x| .
$$

For $x \in W-\{0\}$, the function $V(\lambda) \equiv U(\lambda x)-\Theta^{M}(u) \log |\lambda|$ is bounded above and $\Delta$ subharmonic on $\mathbf{C}$ (since $\left.U\right|_{W}$ is $\Delta$-subharmonic). By Liouville's Theorem $V(\lambda)$ is constant equal to $V(1)=U(x)$. Hence, $V(\lambda)=V(1)$ says that

$$
U(\lambda x)=\Theta^{M}(u) \log |\lambda|+U(x) \quad \forall x \in W, \lambda \in \mathbf{C} .
$$

Setting $y=r e^{i \theta} \in W, x=e^{i \theta}$, this gives the desired result:

$$
U(y)=\Theta^{M}(u) \log |y|+U\left(\frac{y}{|y|}\right) \quad \forall y \in W .
$$

Now the first equality in (7.8) of Part I shows that $\sup _{|x|=1} U(x)=\Theta^{M}(u) \log 1=0$. 


\section{The Strong Uniqueness Theorems.}

To begin we introduce the following concept.

Definition 3.1. We say that $\mathbb{G} \subset G\left(p, \mathbf{R}^{n}\right)$ has the transitivity property if for any two vectors $x, y \in \mathbf{R}^{n}$ there exist $W_{1}, \ldots, W_{k} \in \mathbb{G}$ with $x \in W_{1}, y \in W_{k}$ and $\operatorname{dim}\left(W_{i} \cap W_{i+1}\right)>0$ for all $i=1, \ldots, k-1$.

Note that if any two points $x, y \in \mathbf{R}^{n}$ are contained in $W$ for some $W \in \mathbb{G}$, then, of course, $\mathbb{G}$ has the transitivity property.

THEOREM 3.2. (Strong Uniqueness I). Assume $\mathbb{G}$ has the transitivity property. Then strong uniqueness of tangents holds for all $\mathbb{G}$-plurisubharmonic functions.

Proof. Let $U$ be a tangent at 0 to a $\mathbb{G}$-plurisubharmonic function $u$, and suppose $p \neq 2$. By Theorem 2.1 we know that for every $W \in \mathbb{G}, U(x)=-\frac{\Theta(W)}{\mid x p^{-2}} \forall x \in W$. Thus if $W, W^{\prime} \in \mathbb{G}$ satisfy $\operatorname{dim}\left(W \cap W^{\prime}\right) \geq 1$, then $\Theta(W)=\Theta\left(W^{\prime}\right)$. Hence, by the transitivity property, $\Theta(W)$ is constant on $\mathbb{G}$. Clearly that constant is $\Theta(u, 0)$. When $p=2$ the argument is similar.

\section{Some Examples.}

One can establish the transitivity property for the following sets $\mathbb{G}$, and therefore one has strong uniqueness of tangents for the corresponding $\mathbb{G}$-plurisubharmonic functions.

(a) $\mathbb{G}=G\left(p, \mathbf{R}^{n}\right)$ (p-plurisubharmonic functions) for $p>1$.

(b) $\mathbb{G}=G^{\mathbf{C}}\left(k, \mathbf{C}^{n}\right)$ (complex $k$-plurisubharmonic functions) for $k>1(p=2 k)$.

(c) $\mathbb{G}=G^{\mathbf{H}}\left(k, \mathbf{H}^{n}\right)$ (quaternionic $k$-plurisubharmonic functions) for $k>1(p=4 k)$.

(d) $\mathbb{G}=$ ASSOC (Associative subharmonic functions in $\left.\mathbf{R}^{7}\right)(p=3)$.

(e) $\mathbb{G}=$ COASSOC (Coassociative subharmonic functions in $\left.\mathbf{R}^{7}\right)(p=4)$.

(f) $\mathbb{G}=$ CAYLEY (Cayley subharmonic functions in $\left.\mathbf{R}^{8}\right)(p=4)$.

(g) $\mathbb{G}=$ LAG (Lagrangian subharmonic functions in $\left.\mathbf{C}^{n}\right)(p=n)$.

(h) $\mathbb{G}=\mathrm{ISO}_{\mathrm{p}}\left(p\right.$-isotropic subharmonic functions in $\left.\mathbf{C}^{n}\right)$.

Note 3.3. In the three cases: $G\left(1, \mathbf{R}^{n}\right)$ (i.e., $\left.F=\mathcal{P}\right), G\left(1, \mathbf{C}^{n}\right)$ (i.e., $\left.F=\mathcal{P}^{\mathbf{C}}\right)$, and $G\left(1, \mathbf{H}^{n}\right)$ (i.e., $F=\mathcal{P}^{\mathbf{H}}$ ), strong uniqueness fails. In Section 5 the possible tangents in these cases are completely characterized. In the convex case uniqueness of tangents holds, which of course is classical. In the complex case, uniqueness fails. This is due to Kiselman $[\mathrm{K}]$.

Strong uniqueness in cases (a), (b) and (c) above also follows from Theorem 10.1 in Part I. However, the others do not. 
THEOREM 3.3. (The Transitivity Theorem). Fix $p \geq 2$ and $n \geq 3$.

(a) Every compact $S U(n)$-invariant subset $\mathbb{G} \subset G^{\mathbf{R}}\left(p, \mathbf{C}^{n}\right)$ except $\mathcal{P}^{\mathbf{C}}$ has the transitivity property.

(b) Every compact $S p(n) \cdot \operatorname{Sp}(1)$-invariant subset $\mathbb{G} \subset G^{\mathbf{R}}\left(p, \mathbf{H}^{n}\right)$ with three exceptions has the transitivity property. The exceptions are the sets of real p-planes which lie in a quaternion line for $p=2,3,4$. When $p=4$ this is $\mathcal{P}^{\mathbf{H}}$

(c) For $p \geq 5$, every compact $S p(n)$-invariant subset $\mathbb{G} \subset G^{\mathbf{R}}\left(p, \mathbf{H}^{n}\right)$ has the transitivity property.

Proof of (a). When $p \geq 3$ there is a simple argument, which we give first. Let $W \subset \mathbf{C}^{n}$ be a real 3-plane and consider the orbit $\mathrm{SU}(n) \cdot W \subset G^{\mathbf{R}}\left(3, \mathbf{C}^{n}\right)$.

Lemma 3.4. Given any unit vector $x \in \mathbf{C}^{n}$ and any unit vector $e \perp \operatorname{span}\{x, J x\}$, there exists $V \in \mathrm{SU}(n) \cdot W$ with $x, e \in V$.

Proof. Clearly there exists $W^{\prime} \in \mathrm{SU}(n) \cdot W$ with $x \in W^{\prime}$. Let $H=\{x, J x\}^{\perp}$. Then $\operatorname{dim}_{\mathbf{R}}\left(W^{\prime} \cap H\right) \geq 1$ so there exists a unit vector $e_{0} \in W^{\prime} \cap H$. Thus $W^{\prime}=\operatorname{span}\left\{x, v, e_{0}\right\}$ for a unit vector $v \perp x, e_{0}$.

Choose $g \in \mathrm{SU}(n)$ such that $g x=x$ and $g\left(e_{0}\right)=e$. This is possible since $\mathrm{SU}(n-1) \equiv$ $\{g: g x=x\}$ acts transitively on the unit sphere in $\mathbf{C}^{n-1}$ for $n>2$. Set $V=g\left(W^{\prime}\right)=$ $\operatorname{span}\{x, g v, e\}$.

Corollary 3.5. For any real 3-dimensional subspace $W \subset \mathbf{C}^{n}$, the set $\mathrm{SU}(n) \cdot W$ has the transitivity property. Consequently, any compact $\mathrm{U}(n)$-invariant subset $\mathbb{G} \subset G^{\mathbf{R}}\left(p, \mathbf{C}^{n}\right)$ for $p \geq 3$ has the transitivity property.

Proof. Given non-zero vectors $x, y \in \mathbf{C}^{n}$, choose a unit vector $e$ with $e \perp x, J x, y, J y$. By Lemma 3.4 there exist $W_{x}, W_{y} \in \mathrm{SU}(n) \cdot W$ with $x, e \in W_{x}$ and $y, e \in W_{y}$. Thus, $\mathrm{SU}(n) \cdot W$ has the transitivity property. The second assertion follows immediately.

This leaves the case where $p=2$.

Lemma 3.6. Given a real 2-plane $W \subset \mathbf{C}^{n}$ and any (real) orthonormal basis $x, v$ of $W$, the number $|\langle J x, v\rangle| \equiv \cos \theta$ is a complete invariant of the orbit

$$
\mathbb{G} \equiv \mathrm{SU}(n) \cdot W \subset G^{\mathbf{R}}\left(2, \mathbf{C}^{n}\right) .
$$

Proof. Suppose $y, w$ are orthonormal with $|\langle J y, w\rangle|=\cos \theta$. There exists $g \in \mathrm{SU}(n)$ with $g x=y$, so we may assume $y=x$. By changing the sign of (say) $v$ if necessary, we may assume $\langle J x, v\rangle=\langle J x, w\rangle$. Now $v=\langle v, J x\rangle J x+v_{0}$ and $w=\langle w, J x\rangle J x+w_{0}$, where $v_{0}$ and $w_{0}$ are orthogonal to $x, J x$. Now since $n \geq 3$, there exists $g^{\prime} \in \mathrm{SU}(n-1) \equiv\{g: g x=x\}$, as above, so that $g^{\prime}\left(v_{0}\right)=w_{0}$, and therefore $g^{\prime}(v)=w$.

Part (a) for $p=2$ is a consequence of the following.

Proposition 3.7. If $W \in G^{\mathbf{R}}\left(2, \mathbf{C}^{n}\right)$ is not a complex line, then the orbit $\mathrm{U}(n) \cdot W$ has the transitivity property. 
Proof. Fix a unit vector $x \in \mathbf{C}^{n}$ and consider the set

$$
B_{x} \equiv\left\{v \in \mathbf{C}^{n}:|v|=1 \text { and }\langle J x, v\rangle=\cos \theta\right\}
$$

By assumption the invariant $\cos \theta \neq 1$. Hence this set is a geodesic ball in $S^{2 n-1}$ of intrinsic radius $0<\theta<\pi$ about the point $J x$.

Now suppose $y \in \mathbf{C}^{n}$ is another unit vector with the property that $\partial B_{x} \cap \partial B_{y} \neq \emptyset$, and choose $v \in \partial B_{x} \cap \partial B_{y}$. Then $x \in \operatorname{span}\{x, v\}$ and $y \in \operatorname{span}\{y, v\}$ and by Lemma 3.6 both $\operatorname{span}\{x, v\}$ and $\operatorname{span}\{y, v\}$ lie in $\mathbb{G}$.

In the event that $\partial B_{x} \cap \partial B_{y}=\emptyset$, we can find a sequence of points $x=x_{0}, x_{1}, x_{2}, \ldots, x_{N}=$ $y$ such that

$$
\partial B_{x_{k-1}} \cap \partial B_{x_{k}} \neq \emptyset \quad \text { for } k=1, \ldots, N .
$$

This completes the proof of (a)

Proof of (c). This closely follows the arguments given in Lemma 3.4 and Corollary 3.5, and is omitted.

Proof of (b). Let $W \subset \mathbf{H}^{n}$ be a real 2-plane, and choose an orthonormal basis $\{x, v\}$ of $W$. Let $\pi_{x}^{\perp}$ denote orthogonal projection onto $(\mathbf{H} x)^{\perp} \subset \mathbf{H}^{n}$.

Lemma 3.8. The norm $\left|\pi_{x}^{\perp}(v)\right|^{2}$ is independent of the choice of orthonormal basis $\{x, v\}$ for $W$, and it is a complete invariant for the action of $\operatorname{Sp}(n) \cdot \operatorname{Sp}(1)$ acting on the Grassmannian $G^{\mathbf{R}}\left(2, \mathbf{H}^{n}\right)$.

Proof. Let $e_{0}=1, e_{1}, e_{2}, e_{3}$ be an orthonormal basis of $\mathbf{H}$. Then $\left|\pi_{x}^{\perp}(v)\right|^{2}=1-\langle x, v\rangle^{2}-$ $\sum_{j=1}^{3}\left\langle v, e_{j} x\right\rangle^{2}=1-\sum_{j=1}^{3}\left\langle v, e_{j} x\right\rangle^{2}$. Now let $x^{\prime}=x \cos \theta+v \sin \theta$ and $v^{\prime}=-x \sin \theta+v \cos \theta$ be another orthonormal basis of $W$. Using the fact that $\left\langle v, e_{j} x\right\rangle=-\left\langle e_{j} v, x\right\rangle$, one computes that $\left\langle v^{\prime}, e_{j} x^{\prime}\right\rangle=\left\langle v, e_{j} x\right\rangle \cos ^{2} \theta-\left\langle e_{j} v, x\right\rangle \sin ^{2} \theta=\left\langle v, e_{j} x\right\rangle$ for $j=1,2,3$. This proves the independence of the choice of orthonormal basis.

Now suppose we have 2-planes with o.n. bases $W=\operatorname{span}\{x, v\}$ and $W^{\prime}=\operatorname{span}\left\{x^{\prime}, v^{\prime}\right\}$. Then there exists $g \in \operatorname{Sp}(n)$ with $g(x)=x^{\prime}$, so we may assume that $x=x^{\prime}$. Let $\pi_{x}$ denote orthogonal projection onto the quaternion line $\mathbf{H} x$. The $\operatorname{subgroup}$ of $\operatorname{Sp}(n) \cdot \operatorname{Sp}(n)$ which maps $\mathbf{H} x$ to itself is transitive on all real 2-planes in $\mathbf{H} x$, in fact it contains an $\mathrm{SO}(4)$ subgroup acting standardly on $\mathbf{H} x=\mathbf{R}^{4}$. Thus there is an element in this subgroup which fixes $x$ and maps $\pi_{x} v$ to $\pi_{x} v^{\prime}$ (since they are orthogonal to $x$ and have the same length). Now since $\operatorname{Sp}(n-1) \equiv\{g \in \operatorname{Sp}(n): g(x)=x\}$ acts transitively on the unit sphere in $(\mathbf{H} x)^{\perp}$, it contains an element which maps $\pi_{x}^{\perp} v$ to $\pi_{x}^{\perp} v^{\prime}$.

Proposition 3.9. Let $W$ be a real 2-plane in $\mathbf{H}^{n}$ which is not contained in a quaternion line. Then the orbit $\mathbb{G} \equiv \operatorname{Sp}(n) \cdot \operatorname{Sp}(1) W$ has the transitivity property.

Proof. Let $W=\operatorname{span}\left\{x_{0}, v_{0}\right\}$ as above. By assumption the invariant $\sin ^{2} \theta \equiv\left|\pi_{x_{0}}^{\perp} v_{0}\right|^{2} \neq 0$. Fix a unit vector $x \in \mathbf{H}^{n}$. By Lemma 3.8 the set of 2-planes in $\mathbb{G}$ which contain $x$ is

$\Sigma_{x} \equiv\{W \in \mathbb{G}: x \in W\}=\left\{\operatorname{span}\{x, v\}:\left|\pi_{x}^{\perp} v\right|^{2}=\sin ^{2} \theta\right\} \cong\left\{v \in S^{4 n-1}:\left|\pi_{x}^{\perp} v\right|^{2}=\sin ^{2} \theta\right\}$.

This is the real hypersurface of points in $S^{4 n-1}$ at constant distance $\theta$ from the geodesic 2-sphere $S_{x}^{2} \equiv\{e \cdot x: e \in \operatorname{ImH}$ and $|e|=1\}$. Now it is straightforward to see that 
$\Sigma_{x} \cap \Sigma_{y} \neq \emptyset$ for all $y$ sufficiently close to $x$. By homogeneity the measure of closeness is independent of $x$. The transitivity property follows.

Assertion (b) now follows, and the proof of Theorem 3.3 is complete.

Theorem 3.3 implies that nearly every $\mathrm{SU}(n)$ - or $\operatorname{Sp}(n)$-invariant set $\mathbb{G}$ has the transitivity property. Among the geometrically interesting examples are the sets of Lagrangian and, more generally, isotropic planes in $\mathbf{C}^{n}$ (see Example A.4). Here are further examples.

Example 3.10. (Cauchy-Riemann Sets). Fix integers $1 \leq m$ and $p>2 m$, and define

$$
\mathbb{G}=\left\{W \in G^{\mathbf{R}}\left(p, \mathbf{C}^{n}\right): \operatorname{dim}_{\mathbf{C}}(W \cap J W) \geq m\right\}
$$

Closely related is the set

$$
\mathbb{G}_{0}=\left\{V \oplus L \in G^{\mathbf{R}}\left(2 m+\ell, \mathbf{C}^{n}\right): V=J V, L \perp J L \text { and } \operatorname{dim}_{\mathbf{C}} V=m\right\}
$$

Notice that $\mathbb{G}_{0}$-submanifolds have constant CR-rank $\equiv m$, and $\mathbf{G}$-submanifolds have CRrank $\geq m$.

Example 3.11. (Quaternionic Isotropic and Cauchy-Riemann Sets). In $\mathbf{H}^{n}$ we have the sets of isotropic $p$-planes $(p \leq n)$

$$
\mathrm{ISO}_{p}^{\mathbf{H}} \equiv\left\{W \in G^{\mathbf{R}}\left(p, \mathbf{H}^{n}\right): I w, J w, K w \perp W \forall w \in W\right\}
$$

There are also quaternionic analogues of the Cauchy -Riemann sets given in Example 3.10.

THEOREM 3.12. (Strong Uniqueness II). Fix $p \geq 2$ and $n \geq 3$. Then strong uniqueness of tangents to $\mathbb{G}$-plurisubharmonic functions holds for:

(a) Every compact $S U(n)$-invariant subset $\mathbb{G} \subset G^{\mathbf{R}}\left(p, \mathbf{C}^{n}\right)$ except $\mathcal{P}^{\mathbf{C}}$,

(b) Every compact $S p(n) \cdot \operatorname{Sp}(1)$-invariant subset $\mathbb{G} \subset G^{\mathbf{R}}\left(p, \mathbf{H}^{n}\right)$ with three exceptions, namely the sets of real $p$-planes which lie in a quaternion line for $p=2,3,4$ (when $p=4$ this is $\mathcal{P}^{\mathbf{H}}$ ),

(c) For $p \geq 5$, every compact $S p(n)$-invariant subset $\mathbb{G} \subset G^{\mathbf{R}}\left(p, \mathbf{H}^{n}\right)$.

Proof. This is an immediate consequence of Theorems 3.2 and 3.3 above. 


\section{Homogeneous F-Subharmonics.}

We begin by computing the formula for the second derivative $D_{x}^{2} u$ of a function $u$, which is homogeneous of degree $m$, in terms of its restriction $\left.g \equiv u\right|_{S^{n-1}}$ to the unit sphere $S^{n-1} \subset \mathbf{R}^{n}$. For our application it is useful to replace $m$ by $p \equiv-m+2($ or $m=-(p-2))$ so that:

$$
u(x)=\frac{1}{|x|^{p-2}} g\left(\frac{x}{|x|}\right) .
$$

Remark 4.1. $(p=2)$. In the special case $p=2$ the natural extension of $g$ is given by $u(x)=\Theta \log |x|+g\left(\frac{x}{|x|}\right)$. This choice is consistent with the Riesz kernels and with classical pluripotential theory. The formulas computed below, when $p=2$, only apply to the special case $\Theta=0$. However, they also apply directly to give the corresponding formulas in the general case. This is discussed in Remark 5.3.

Let $\operatorname{Hess}_{\sigma} g$ denote the riemannian hessian of $g$ at a point $\sigma=x /|x| \in S^{n-2}$. Using the orthogonal decomposition

$$
\mathbf{R}^{n}=T_{\sigma}\left(\mathbf{R}^{n}\right)=T_{\sigma}\left(S^{n-1}\right) \oplus N_{\sigma}\left(S^{n-1}\right)
$$

the quadratic form $\operatorname{Hess}_{\sigma} g$ on $T_{\sigma}\left(\mathbf{R}^{n}\right)$ can be considered to be a quadratic form on $\mathbf{R}^{n}$ (whose null space contains $N_{\sigma}\left(S^{n-1}\right)$ ). Also, the tangential derivative $D_{\sigma} g=d g$ at $\sigma$ can be considered a vector in $\mathbf{R}^{n}$. Then

$$
|x|^{p} D_{x}^{2} u=\operatorname{Hess}_{\sigma} g-(p-2) g P_{x^{\perp}}-(p-1)\left(\sigma \circ D_{\sigma} g\right)+(p-2)(p-1) g P_{x}
$$

where on the right hand side, $J^{2} g \equiv(g, D g$, Hess $g)$, the riemannian 2-jet of $g \in C^{2}\left(S^{n-1}\right)$, is evaluated at the point $\sigma=x /|x| \in S^{n-1}$.

Proof of (4.3). One computes that $D\left(\frac{1}{|x|^{p-2}}\right)=-\frac{p-2}{|x|^{p-1}} \frac{x}{|x|}$, and from Lemma 2.1, we have that

$$
|x|^{p} D^{2}\left(\frac{1}{|x|^{p-2}}\right)=-(p-2) P_{[x]^{\perp}}+(p-2)(p-1) P_{[x]} .
$$

Define $\widetilde{g}(x)=g(x /|x|)$ for $x \in \mathbf{R}^{n}-\{0\}$. Then direct calculation shows that $D \widetilde{g}(x) \cong$ $(1 /|x|) D_{\sigma} g$ and that

$$
D_{x}^{2} \widetilde{g} \cong \frac{1}{|x|^{2}}(\operatorname{Hess} g-\sigma \circ D g)
$$

These formulas for the first and second derivatives of the functions $1 /|x|^{p-2}$ and $\widetilde{g}$ yield the formula (4.3) for the second derivative of the product $u$.

Define $\Phi\left(J_{\sigma}^{2} g\right) \in \operatorname{Sym}^{2}\left(\mathbf{R}^{n}\right)$ to be the RHS of (4.3). That is,

$$
\Phi\left(J_{\sigma}^{2} g\right) \equiv \operatorname{Hess}_{\sigma} g-(p-2) g(\sigma) P_{\sigma^{\perp}}-(p-1)\left(\sigma \circ D_{\sigma} g\right)+(p-2)(p-1) g(\sigma) P_{\sigma} .
$$

Then (4.3) says that

$$
|x|^{p} D_{x}^{2} u=\Phi\left(J_{\sigma}^{2} g\right) \quad \text { with } \sigma \equiv \frac{x}{|x|}
$$


In terms of the $2 \times 2$-blocking induced on $\operatorname{Sym}^{2}\left(\mathbf{R}^{n}\right)$ by the decomposition (4.2)

$$
\Phi\left(J_{\sigma}^{2} g\right)=\left(\begin{array}{cc}
\text { Hess } g-(p-2) g I & -(p-1) D g \\
-(p-1) D g^{t} & (p-2)(p-1) g
\end{array}\right)
$$

with RHS evaluated at $\sigma$ (and with $D g$ written as a column vector.)

The formula $(4.3)^{\prime}$ has been proved for $u$ and $g$ related by (4.1) and of class $C^{2}$. This immediately implies the following.

Proposition 4.2. For a cone subequation $F$ and $u(x)=\frac{1}{|x|^{p-2}} g\left(\frac{x}{|x|}\right)$ of class $C^{2}$, $u$ is $F$-subharmonic on $\mathbf{R}^{n}-\{0\} \quad \Longleftrightarrow \quad \Phi\left(J_{x}(g)\right) \in F \quad \forall|x|=1, \quad$ and

$u$ is F-harmonic on $\mathbf{R}^{n}-\{0\} \quad \Longleftrightarrow \quad \Phi\left(J_{x}(g)\right) \in \partial F \quad \forall|x|=1$,

We wish to extend this proposition to include upper semi-continuous functions $u$ and $g$. Note that with $u$ and $g$ related by (4.1), $u$ is upper semi-continuous on $\mathbf{R}^{n}-\{0\}$ if and only if $g$ is upper semi-continuous on $S^{n-1}$.

Lemma 4.3. Given a subset $F \subset \operatorname{Sym}^{2}\left(\mathbf{R}^{n}\right)$, consider the subset

$$
F_{S^{n-1}} \equiv \Phi^{-1}(F)
$$

of the 2-jet bundle $J^{2}\left(S^{n-1}\right)$.

(1) $F$ closed $\Rightarrow \quad F_{S^{n-1}}$ closed.

(2) $F$ satisfies $(P) \quad \Rightarrow \quad F_{S^{n-1}}$ satisfies $(P)$.

(3) $F$ is a cone $\Rightarrow \quad F_{S^{n-1}}$ is a cone bundle,

(4) $F$ is a convex cone $\Rightarrow \quad F_{S^{n-1}}$ is a convex cone bundle.

(5) For any subgroup $H \subset \mathrm{O}(n)$

$$
F \text { is } H \text {-invariant } \quad \Rightarrow \quad F_{S^{n-1}} \text { is } H \text {-invariant }
$$

(6) If $F$ is a cone subequation, i.e., (1), (2) and (3) are true, then the dual

$$
\widetilde{F_{S^{n-1}}}=(\widetilde{F})_{S^{n-1}} \text {. }
$$

(7) Suppose $F$ is a cone subequation with Riesz charactersitic $p$.

(a) If $p>2$, then

$$
F_{S^{n-1}} \text { satisfies }(N) \quad \Longleftrightarrow \quad F \text { is } \mathcal{P}_{p^{-} \text {-monotone. }}
$$

(b) If $1 \leq p<2$, then ( $N$ ) fails for $F_{S^{n-1}}$.

Before proving this lemma we state the main result. But first consider the following.

Example 4.4. Let $F \equiv\{A: \operatorname{tr} A \geq 0\}=\Delta$ be the standard Laplacian on $\mathbf{R}^{n}$. Then by $(4.6)^{\prime} F_{S^{n-1}}$ is the linear subequation $L g \geq 0$ on $S^{n-1}$, where

$$
L g \equiv \operatorname{tr} \Phi(J(g))=\Delta_{S^{n-1}} g-(n-p)(p-2) g .
$$

Note that $L$ satisfies (N) if $2<p \leq n$.

Now the extension of Proposition 4.2 to include u.s.c. functions $u$ and $g$ can be stated as follows. 
THEOREM 4.5. $(p \neq 2)$. Suppose that $F \subset \operatorname{Sym}^{2}\left(\mathbf{R}^{n}\right)$ is a cone subequation. If $u$ and $g$ are upper semi-continuous functions related by (4.1), then

$$
\begin{aligned}
& u \text { is } F \text {-subharmonic on } \mathbf{R}^{n}-\{0\} \quad \Longleftrightarrow \quad g \text { is } F_{S^{n-1} \text {-subharmonic on } S^{n-1} \text {, and }} \\
& u \text { is } F \text {-harmonic on } \mathbf{R}^{n}-\{0\} \quad \Longleftrightarrow \quad g \text { is } F_{S^{n-1}} \text {-harmonic on } S^{n-1} \\
& \Longleftrightarrow \quad g \text { is } F_{S^{n-1}-\text { subharmonic on } S^{n-1} \text { and }} \\
& -g \text { is } \widetilde{F}_{S^{n-1}} \text {-subharmonic on } S^{n-1} \text {. }
\end{aligned}
$$

In the applications typically $u$ is $F$-subharmonic across 0 . This imposes an additional condition on $g$.

Proposition 4.6. Suppose $F$ has Riesz characteristic $1 \leq p<\infty, p \neq 2$, and that $u(x) \equiv \frac{1}{|x|^{p-2}} g\left(\frac{x}{|x|}\right)$ is $F$-subharmonic on $\mathbf{R}^{n}$ across 0 . Let $\Theta \equiv \Theta^{M}(u, 0)$ be the density of $u$ at 0 . Then:

(1) $g$ is $F_{S^{n-1-s u b h a r m o n i c} \text { on } S^{n-1} \text {, and }}$

(2) if $2<p<\infty$, then $\sup _{S^{n-1}} g=-\Theta \leq 0$, while

(3) if $1 \leq p<2$, then $\sup _{S^{n-1}} g=\Theta \geq 0$.

Proof. Note that $K(1)=-1$ for $2<p<\infty$, while $K(1)=1$ for $1 \leq p<2$. Now Proposition 5.6 can be used to compute $\Theta^{M}(u, 0)$.

Remark 4.7. If $F$ is $\mathcal{P}_{p}$-monotone and $2<p$, then a converse is true, since $(2) \Rightarrow u$ is locally bounded above across 0 , in which case the singularity at 0 is removable by results in [HL9]. Thus (1) and (2) imply that $u$ is $F$-subharmonic on $\mathbf{R}^{n}$.

Proof of Lemma 4.3. Formula (4.6)' shows that

$$
\begin{gathered}
\Phi: J^{2}\left(S^{n-1}\right) \longrightarrow S^{n-1} \times\left.\operatorname{Sym}^{2}\left(\mathbf{R}^{n}\right) \equiv \operatorname{Sym}^{2}\left(\mathbf{R}^{n}\right)\right|_{S^{n-1}} \\
\quad \text { is an } \mathrm{O}(\mathrm{n}) \text { - equivariant bundle map, }
\end{gathered}
$$

and, in fact when $(p-1)(p-2) \neq 0$, it is a bundle isomorphism. From this the implications $(1),(3),(4)$ and $(5)$ are obvious. To prove (2) note that with

$$
J_{\sigma}\left(S^{n-1}\right) \cong \mathbf{R} \times T_{\sigma}^{*} S^{n-1} \times \operatorname{Sym}^{2}\left(T_{\sigma}^{*} S^{n-1}\right),
$$

we have

$$
\Phi(0,0, P)=\left(\begin{array}{ll}
P & 0 \\
0 & 0
\end{array}\right) \in \mathcal{P} \quad \text { if } P \geq 0 .
$$

To prove (6) note that (4.8) implies that $\Phi^{-1}(\operatorname{Int} F)=\operatorname{Int} \Phi^{-1}(F)$, and that the fibres of $\operatorname{Int} \Phi^{-1}(F)$ are the fibres of $\Phi^{-1}(\operatorname{Int} F)$. For $(7)$ note that by (4.6)

$$
\Phi(J+(-r, 0,0))=\Phi(J)+r(p-2)\left(P_{e^{\perp}}-(p-1) P_{e}\right) .
$$

Hence, for $p>2, F_{S^{n-1}}$ satisfies $(\mathrm{N}) \Longleftrightarrow F$ is $\mathcal{P}_{p^{-m o n o t o n e, ~ w h i l e ~ f o r ~} 1<p<2, F_{S^{n-1}}}$ doesn't satisfy $(\mathrm{N})$. 
Proof of Theorem 4.5. The implications $\Rightarrow$ are easy since a test function $\psi$ for $g$ at $\sigma$ induces a test function $\varphi(x) \equiv \frac{1}{|x|^{p-2}} \psi\left(\frac{x}{|x|}\right)$ for $u$ at $\sigma$.

To prove the reverse implications we fix a point $x_{0}$ which we may assume to be of the form $x_{0}=(\rho, 0, \ldots, 0)$ for $\rho>0$. We then choose the local coordinate $y$ on the sphere about $(1,0, \ldots, 0)$ given by $\Psi(y)=(1, y) /|(1, y)|$ for $|y|<1$. Setting $t=r-\rho$ gives local coordinates $(t, y)$ about $x_{0}$ on $\mathbf{R}^{n}$ with $(0,0)$ corresponding to $x_{0}$.

Under this coordinate change a function of the form $|x|^{2-p} g\left(\frac{x}{|x|}\right)$ becomes $(\rho+t)^{2-p} \gamma(y)$. To complete the proof it will suffice to prove the following lemma.

Lemma 4.8. Suppose $\varphi(t, y))$ is a strict quadratic test function for the function $u(t, y)=$ $(\rho+t)^{2-p} \gamma(y)$ at $(0,0)$. Then there exists a smooth test function $\psi(y)$ for $\gamma(y)$ at 0 in $\mathbf{R}^{n-1}$ such that

$$
(\rho+t)^{2-p} \gamma(y) \leq(\rho+t)^{2-p} \psi(y) \leq \varphi(t, y) \quad \text { near }(0,0) .
$$

Proof. We can assume that $\gamma(0)=0$. By assumption $\varphi$ is a strict test function of the form

$$
\varphi(t, y)=p t+\langle q, y\rangle+a t^{2}+2\langle b, y\rangle t+\langle C y, y\rangle .
$$

Setting $y=0$ gives $0=u(t, 0)<p t+a t^{2}$ and therefore

$$
p=0 \quad \text { and } \quad a>0 .
$$

We now have

$$
(\rho+t)^{2-p} \gamma(y) \equiv u(t, y) \leq \varphi(t, y)=a t^{2}+2\langle b, y\rangle t+k \equiv Q_{y}(t)
$$

where

$$
k=k(y) \equiv\langle q, y\rangle+\langle C y, y\rangle .
$$

For fixed $\delta>0$ small we define

$$
\psi(y) \equiv \inf _{|t| \leq \delta} \frac{1}{(t+\rho)^{2-p}} Q_{y}(t) .
$$

Then on $\{|t| \leq \delta\}$ we have

(1) $u(t, y) \leq(t+\rho)^{2-p} \psi(y) \quad$ (because $g(y) \leq \psi(y)$ ), and

(2) $(t+\rho)^{2-p} \psi(y) \leq \varphi(t, y)$.

It remains to show that $\psi(t)$ is smooth when $\delta$ is taken sufficiently small. One calculates that $t$ is a critical point of the function $t \mapsto(t+\rho)^{p-2} Q_{y}(t)$ in the range $t+\rho>0$ if and only if

$$
A t^{2}+2 B t+C=0
$$

where

$$
A=a p, \quad B=\rho a+(p-1)\langle b, y\rangle, \quad C=2 \rho\langle b, y\rangle+(p-2) k(y) .
$$


When $y=0$, we have that $a p t^{2}+2 \rho a t=0$ which happens iff

$$
t=0 \quad \text { or } \quad t=-\frac{2 \rho}{p}
$$

We choose $\delta<<2 \rho / p$ to rule out the second possibility. The roots $t_{1}(y)$ and $t_{2}(y)$ of (4.9) with $t_{1}(0)=0$ are two smooth functions of $y$ in a neighborhood of 0 .

It remains to show that $\psi(y)=t_{1}(y)$. Since $t_{1}(y)$ and $t_{2}(y)$ are the critical points of $(t+\rho)^{p-2} Q_{y}(t)$, this means we must show that $\inf _{|t| \leq \delta}(t+\rho)^{p-2} Q_{y}(t)$ is not assumed for $t= \pm \delta$. One checks that this is true for $y=0$ and therefore for all sufficiently small $y$.

\section{Tangents to Convex, C-Plurisubharmonic, and H-Plurisubharmonic Functions.}

We now give a brief discussion of three geometric cases where uniqueness of tangents does not hold. These are the convex functions (where $\mathbb{G}=G\left(1, \mathbf{R}^{n}\right)$ ), the classical complex plurisubharmonic functions (where $\mathbb{G}=G\left(1, \mathbf{C}^{n}\right)$ ), and the quaternionic plurisubharmonic functions (where $\mathbb{G}=G\left(1, \mathbf{H}^{n}\right)$ ). The results in the first case follow from classical convex analysis $[R]$. Those in the complex case are due to Kiselman $[K]$. The results in the quaternionic case are new.

\section{Tangents to Convex Functions}

Suppose $u$ is a convex function defined in a neighborhood of 0 in $\mathbf{R}^{n}$, or equivalently, $u$ is $\mathcal{P}=F\left(G\left(1, \mathbf{R}^{n}\right)\right)$-subharmonic. The Riesz characteristic of this subequation is 1 , and the appropriate homotheties are

$$
u_{r}(x) \equiv \frac{1}{r}(u(r x)-u(0)), \quad r>0
$$

Tangents are unique. In fact,

$$
u_{r} \downarrow U \equiv \lim _{r \downarrow 0} u_{r} \quad \text { uniformly on compact subsets of } \mathbf{R}^{n} \text {. }
$$

This is easy to see geometrically. The mappings $\Psi_{r}: \mathbf{R}^{n+1} \rightarrow: \mathbf{R}^{n+1}$ given by $(x, t) \mapsto$ $\frac{1}{r}(x, t-u(0))$ carry the epigraph of $u$ to the epigraph of $u_{r}$. Convexity implies that for $0<r<s, \operatorname{epi}\left(u_{r}\right) \supset \operatorname{epi}\left(u_{s}\right)$. The epigraphs epi $\left(u_{r}\right)$ increase to epi $(U)$, that is, the functions $u_{r}$ decrease to $U$. The local uniform convergence follows.

Tangents are homogeneous of degree 1, that is,

$$
U_{r}(x)=U(x), \text { i.e., } U(r x)=r U(x) \text {. }
$$

This is immediate since tangents are unique. 
The subdifferential of $u$ at 0 , denoted $(\partial u)(0)$, is the set of $p \in \mathbf{R}^{n}$ such that $u(x)-$ $u(0) \geq\langle p, x\rangle$ for $|x|$ small. It is easy to see that $(\partial u)(0)$ is a non-empty compact convex set. Now the unique tangent function $U$ to $u$ at 0 is related to the subdifferential by

$$
U(x)=\sup _{p \in(\partial u)(0)}\langle p, x\rangle
$$

Note that $p \in(\partial u)(0) \Longleftrightarrow \frac{1}{r}(u(r x)-u(0)) \geq \frac{1}{r}\langle p, r x\rangle=\langle p, x\rangle$, and hence

$$
\begin{gathered}
p \in(\partial u)(0) \quad \Longleftrightarrow \quad p \in\left(\partial u_{r}\right)(0) \quad \Longleftrightarrow p \in(\partial U)(0) \\
\text { and } U(x) \geq\langle p, x\rangle \quad \forall p \in(\partial u)(0) .
\end{gathered}
$$

Finally we show that

$$
\Theta^{S}(u, x)=0 \quad \Longleftrightarrow \quad u \text { is differentiable at } x .
$$

(When this holds, $\Theta^{M}(u, x)=\left|D_{x} u\right|$.) Both assertions in (5.5) remain unchanged if we subtract an affine function from $u$. By subtracting a supporting affine function we may assume that $u \geq 0$ and $u(x)=0$. Then by (5.1) $U$ is differentiable at $x$ if and only if the tangent $U$ at $x$ is $\equiv 0$. Now $\Theta^{S}(U, 0)=\Theta^{S}(u, x)$, so if $\Theta^{S}(u, x)=0$, then the homogeneity of $U$ implies that $f_{S} U(t \sigma) d \sigma=\Theta^{S}(U, 0)=0$ for all $t \geq 0$. However, $U \geq 0$ since $u(r x) / r \downarrow U$ and $u \geq 0$, and so $U=0$. Conversely, if $U=0$, then since $u(r x) / r$ converges uniformly to $U=0, u$ is differentiable at $\mathrm{x}$ with $D_{x} u=0$.

\section{Homogeneous Convex Functions}

Every convex function $U$ which is homogeneous of degree 1 is, of course, the unique tangent to itself at 0 . By subtracting off an affine function, one can always assume that

$$
U \geq 0 \quad \text { and } \quad U(0)=0 .
$$

Such functions are classically understood. Rewrite $U$ as $U(x)=\|x\|$. Then

$$
\|\lambda x\|=\lambda\|x\| \quad \forall \lambda \geq 0, x \in \mathbf{R}^{n} \quad \text { and } \quad\|x+y\| \leq\|x\|+\|y\|,
$$

that is, $\|\bullet\|$ is a semi-norm on $\mathbf{R}^{n}$ (not necessarily balanced). By (5.3) the unit ball $\|p\|^{*} \leq 1$ in the dual norm $\|\bullet\|^{*}$ is the subdifferential $(\partial U)(0)$.

Let $U(x)$ be a $C^{2}$-function which is homogeneous of degree 1, i.e., $U(x)=|x| g\left(\frac{x}{|x|}\right)$ where $\left.g \equiv U\right|_{S^{n-1}}$. Then formula (4.3) with $p=1$ states that

$$
D_{x}^{2} U=\frac{1}{|x|}\left(\operatorname{Hess}_{e} g+g(e) P_{e^{\perp}}\right) \quad \text { where } e=\frac{x}{|x|} .
$$

That is, $D_{x}^{2} U$ is the pull back of the quadratic form $\operatorname{Hess}_{e} g+g I$ on the tangent space $T_{e} S^{n-1}$ to the sphere using the splitting $\mathbf{R}^{n}=T_{e} S^{n-1} \oplus \mathbf{R} \cdot e$. Theorem 4.5 gives the following. 
Proposition 5.1. Let $g \in C\left(S^{n-1}\right)$ be a continuous non-negative function on the sphere $S^{n-1}$, and extend $g$ to a homogeneous function $U(x) \equiv|x| g\left(\frac{x}{|x|}\right)$ of degree 1 on $\mathbf{R}^{n}$. Then $U$ is convex on $\mathbf{R}^{n} \quad \Longleftrightarrow \quad$ Hess $g+g I \geq 0$ (in the viscosity sense) on $S^{n-1}$.

When $n=2$, this is the subquation $g^{\prime \prime}(\theta)+g(\theta) \geq 0$ on $S^{1}$. Note that the negativity condition $(\mathrm{N})$ fails.

Summary. Tangents are unique; strong uniqueness fails; but tangents can be characterized by (5.6) or Proposition 5.1.

\section{Tangents to Plurisubharmonic Functions in $\mathbf{C}^{n}$}

In 1988 Christer Kiselman proved that tangents to plurisubharmonic functions are not unique. In fact he completely characterized the subsets of $L_{\mathrm{loc}}^{1}\left(\mathbf{C}^{n}\right)$ which arise as the tangent sets to psh functions. (See Theorem 4.1 in $[\mathrm{K}]$.) We present those results here.

Since the Riesz characteristic in this case is 2, the appropriate homotheties are

$$
u_{r}(x) \equiv u(r x)-\sup _{B_{r}} u, \quad r>0 .
$$

The following is (essentially) one of Kiselman's results in $[\mathrm{K}]$. Let

$$
\pi: \mathbf{C}^{n}-\{0\} \rightarrow \mathbf{P}_{\mathbf{C}}^{n-1}
$$

denote the standard map to complex projective space, and let $\omega$ denote the standard Kähler form on $\mathbf{P}_{\mathbf{C}}^{n-1}$ so that $\pi^{*} \omega=i \partial \bar{\partial} \log |z|$ on $\mathbf{C}^{n}-\{0\}$.

Proposition 5.2. Suppose $U$ is a tangent to a plurisubharmonic function $u$ defined in a neighborhood of the origin in $\mathbf{C}^{n}$. Then $U$ is of the form

$$
U(x)=\Theta \log |x|+\pi^{*} g \quad \text { with } \quad g \in \operatorname{USC}\left(\mathbf{P}_{\mathbf{C}}^{n-1}\right)
$$

where

(i) $\Theta \equiv \Theta^{M}(u)$ is the (maximum) density of $u$ at 0 ,

(ii) $g$ is $\Theta$-quasi plurisubharmonic on $\mathbf{P}_{\mathbf{C}}^{n-1}$, that is

$$
i \partial \bar{\partial} g+\Theta \omega \geq 0
$$

(iii) and

$$
\sup _{\mathbf{P}_{\mathbf{C}}^{n-1}} g=0
$$

Since only (5.9) is not stated in $[\mathrm{K}]$, we include its straightforward proof. Assume (5.8) has been established, where $g \in \operatorname{USC}\left(\mathbf{P}_{\mathbf{C}}^{n-1}\right)$. Then $i \partial \bar{\partial} U=i \partial \bar{\partial}\left(\Theta \log |x|+\pi^{*} g\right)=$ $\pi^{*}(\Theta \omega+i \partial \bar{\partial} g)$, from which one concludes that

$$
U \text { is plurisubharmonic on } \mathbf{C}^{n}-\{0\} \quad \Longleftrightarrow \quad i \partial \bar{\partial} g+\Theta \omega \geq 0 \text { on } \mathbf{P}_{\mathbf{C}}^{n-1}
$$


Remark 5.3. This result can be deduced from the case $p=2$ in the last section (see Remark 4.1). If

$$
U(x)=\Theta \log |x|+\bar{g}\left(\frac{x}{|x|}\right) \quad \forall x \in \mathbf{C}^{n},
$$

then

$$
D_{x}^{2} U=\frac{1}{|x|^{2}}\left(\begin{array}{cc}
\operatorname{Hess}_{e} \bar{g}+\Theta I & -D_{e} \bar{g} \\
-\left(D_{e} \bar{g}\right)^{t} & -\Theta
\end{array}\right)
$$

One can show that the hermitian symmetric part $\left(D_{x}^{2} U\right)_{\mathbf{C}}$ vanishes on $\mathbf{C} e$ and equals $\left(\operatorname{Hess}_{e} \bar{g}\right)_{\mathbf{C}}+\Theta P_{(\mathbf{C} e)^{\perp}}$ on $(\mathbf{C} e)^{\perp}$ (compare the more complicated quaternionic case below). This completes a second proof.

Remark 5.4. Note also that each $u$ is maximal on $\mathbf{C}^{n}-\{0\}$ since its restriction to each complex line through the origin is $\Delta$-harmonic.

Proposition 5.2 characterizes the possible tangent functions to $u$ at 0 . Kiselman's characterization of the possible tangent sets $T_{0} u$ can be stated as follows.

THEOREM 5.5. (C. Kiselman [K]). Suppose that $\Theta \geq 0$ and that $M$ is a nonempty subset of the $\Theta$-quasi-plurisubharmonic functions on $\mathbf{P}_{\mathbf{C}}^{n-1}$ with each element $g \in$ $M$ satisfying (5.10). If $M$ is closed and connected in $L_{\text {loc }}^{1}\left(\mathbf{P}_{\mathbf{C}}^{n-1}\right)$, then there exists a plurisubharmonic function $u$ defined on a neighborhood of the origin in $\mathbf{C}^{n}$ such that

$$
T_{0} u=M .
$$

\section{Homogeneous Quaternionic Harmonics}

The remaining series of ST-invariant geometric cases where strong uniqueness fails is the case of quaternionic plurisubharmonic functions $\left(\mathbb{G}=G^{\mathbf{H}}\left(1, \mathbf{H}^{n}\right)\right.$ in Section 3). Such functions have been studied by S. Alesker and M. Verbitsky in $\left[A_{1}\right],[A V]$, and also by the authors $\left[\mathrm{HL}_{3}\right],\left[\mathrm{HL}_{4}\right]$. Note that in this case the Riesz characteristic is 4 . Let $\pi: \mathbf{H}^{n}-\{0\} \rightarrow \mathbf{P}_{\mathbf{H}}^{n-1}$ denote the standard map to quaternionic projective space

Proposition 5.6. Suppose $U$ is a tangent to a quaternionic plurisubharmonic function $u$ defined in a neighborhood of the origin in $\mathbf{H}^{n}$. Then $U$ is of the form

$$
U(x)=\frac{1}{|x|^{2}} \pi^{*} g \quad \text { with } \quad g \in \operatorname{USC}\left(\mathbf{P}_{\mathbf{H}}^{n-1}\right)
$$

where $g$ satisfies the subequation

$$
\operatorname{Hess}_{\mathbf{H}}(g)-2 g I \geq 0 \quad \text { on } \mathbf{P}_{\mathbf{H}}^{n-1} .
$$

Here $\operatorname{Hess}_{\mathbf{H}}(g)$ is the quaternionic hermitian symmetric part of the riemannian hessian $H=$ Hess $g$ on $\mathbf{P}_{\mathbf{H}}^{n-1}$, defined by

$$
H_{\mathbf{H}}(v, w)=\frac{1}{4}\{H(v, w)+H(I v, I w)+H(J v, J w)+H(K v, K w) \cdot\}
$$


Proof. By Theorem 2.1 we know that $\left.U\right|_{W \cap S^{4 n-1}}$ is constant for every quaternion line $W \subset \mathbf{H}^{n}$. Hence, $\left.U\right|_{W \cap S^{4 n-1}}=\pi^{*} g$ for some $g \in \operatorname{USC}\left(\mathbf{P}_{\mathbf{H}}^{n-1}\right)$. To simplify notation and to make accord with Section 4 , we shall denote $\pi^{*} g$ simply by $g$.

Consider the unit sphere $S^{4 n-1} \subset \mathbf{H}^{n}=\mathbf{R}^{4 n}$. At any $x \in S^{4 n-1}$ we get a decomposition of $\mathbf{R}^{4 n}$ as

$$
\mathbf{R}^{4 n}=\mathcal{H}_{x} \oplus \mathcal{V}_{x} \oplus \mathbf{R} x
$$

where $\mathcal{V}_{x}$ is the tangent space to the fibre at $x$ of the fibration

$$
\pi: S^{4 n-1} \longrightarrow \mathbf{P}_{\mathbf{H}}^{n-1}
$$

and $\mathcal{H}_{x}$ is the orthogonal complement of $\mathcal{V}_{x}$ in $T_{x} S^{4 n-1}$. $\mathcal{H}$ is horizontal for $\pi$ and $\pi_{*}$ maps it isometrically onto $T_{\pi x} \mathbf{P}_{\mathbf{H}}^{n-1}$. It is an $\mathbf{H}$-linear subspace of $\mathbf{H}^{n}$. Note that the radial $\mathbf{H}$-line through $x$ satisfies $\mathbf{H} x=\mathcal{V}_{x} \oplus \mathbf{R} x$.

Let $I, J, K$ be the standard basis for the imaginary quaternions. Then we get a trivialization of $\mathcal{V}$ by the global vector fields:

$$
V_{1}(x)=I(x), \quad V_{2}(x)=J(x), \quad V_{3}(x)=K(x) .
$$

Now we are considering the operator from $(4.6)^{\prime}$

$$
L(g)=\left(\begin{array}{cc}
\operatorname{Hess} g-2 g I & -3 D g \\
-3 D g^{t} & 6 g
\end{array}\right)
$$

written with respect to the splitting $T_{x} S^{4 n-1} \oplus \mathbf{R} x$. We want to compute this for a function $g=\pi^{*} \widetilde{g}$ where $\widetilde{g}: \mathbf{P}_{\mathbf{H}}^{n-1} \rightarrow \mathbf{R}$. More precisely we want to compute the quaternionic hermitian symmetric part:

$$
L_{\mathbf{H}}(g)(v, w) \equiv \frac{1}{4}\{(L g)(v, w)+(L g)(I v, I w)+(L g)(J v, J w)+(L g)(K v, K w)\} .
$$

Our first observation is that

$$
\mathcal{V} \subset \operatorname{Ker}(D g) \quad \text { and } \quad \mathcal{V} \subset \operatorname{Null}(\operatorname{Hess} g) .
$$

Thus if $v \in \mathcal{V}$, then we find that

$$
4 L_{\mathbf{H}}(g)(v, v)=-2 g-2 g-2 g+6 g=0 .
$$

Similarly, if $e=x$ we have $L_{\mathbf{H}}(g)(e, e)=0$, and $L_{\mathbf{H}}(g)(v, e)=0$. Thus $\mathbf{H} \cdot x$ (the radial $\mathbf{H}$-line) lies in the kernel of $L_{\mathbf{H}}(g)$ (cf. (5.17) below).

We now want to compute the spherical hessian $\operatorname{Hess}(g)$. Let $H$ be an invariant horizontal vector field obtained by lifting a vector field $\widetilde{H}$ on $\mathbf{P}_{\mathbf{H}}^{n-1}$ via $\pi$, and let $V$ be a vertical vector field which is a real linear combination of the $V_{j}$ above. Then

$$
0=\mathcal{L}_{V}(H)=[V, H]=\nabla_{V} H-\nabla_{H} V
$$


where $\nabla$ is the riemannian connection on the sphere. Observe now that

$$
\nabla_{H} V=\nabla_{H}(J x)=\left\{\nabla_{H}^{\mathbf{R}^{4 n+4}}(J x)\right\}^{\operatorname{Tan}}=\{(J H)\}^{\text {Tan }}=(J H) \cong V H
$$

where we identify $J \cong V$ via the global identification ((5.15)) above:

$$
\mathcal{V} \cong \operatorname{Im} \mathbf{H}
$$

Now let $H_{1}, H_{2}$ be invariant horizontal vector fields as above. Then

$$
\begin{aligned}
(\text { Hess } g)\left(H_{1}, H_{2}\right) & =H_{1} H_{2} g-\left(\nabla_{H_{1}} H_{2}\right) g \\
& =H_{1} H_{2} g-\left(\nabla_{H_{1}} H_{2}\right)^{\mathcal{H}} g \\
& =\left(\operatorname{Hess}_{\mathbf{H}} g\right)\left(H_{1}, H_{2}\right) \quad \text { lifted to the sphere }
\end{aligned}
$$

We must now compute the $(\mathcal{V}, \mathcal{H})$-component of Hess $g$.

$$
\begin{aligned}
(\operatorname{Hess} g)(H, V) & =H V g-\left(\nabla_{H} V\right) g \\
& =0-\left\langle\left(\nabla_{H} V\right), D g\right\rangle \\
& =-\langle V H, D g\rangle=-\left\langle H, \mu_{V}^{*} D g\right\rangle
\end{aligned}
$$

by ((5.16)) above, where $\mu_{V}$ is the action of the imaginary quaternion $V$ on $\mathcal{H}$ at $x$.

So with respect to the splitting $((5.14))$ we have

$$
L(g)=\left(\begin{array}{ccc}
\operatorname{Hess}^{\mathbf{P}} g-2 g I & -\mu^{*} D g & -3 D g \\
\mu^{*} D g^{t} & -2 g I & 0 \\
-3 D g^{t} & 0 & 6 g
\end{array}\right)
$$

Now we know that

$$
L_{\mathbf{H}}(g)=\left(\begin{array}{ccc}
\operatorname{Hess}_{\mathbf{H}}^{\mathbf{P}} g-2 g I & * & * \\
* & 0 & 0 \\
* & 0 & 0
\end{array}\right)
$$

It remains only to compute the $\mathbf{H}$-symmetric part of the *'s.

Consider a vector $(a, b) \in \mathcal{H} \oplus(\mathcal{V} \oplus \mathbf{R} x)=\mathcal{H} \oplus \mathcal{H}^{\perp}$. We want to look at the term $\left\langle L_{\mathbf{H}} a, b\right\rangle$. This is

$$
\left\langle L_{\mathbf{H}} a, b\right\rangle=\frac{1}{4}\{\langle L a, b\rangle+\langle L I a, I b\rangle+\langle L J a, J b\rangle+\langle L K a, K b\rangle .\}
$$

This is the trace of a quadratic form on $\mathbf{H}=\mathbf{R}^{4}$, and it can be expressed with respect to any orthonormal basis. As a result we may assume that $b=x \equiv e$ and $a \in \mathcal{V}$.

$$
\langle L a, b\rangle=\langle-3(D g) a, e\rangle=-3\langle(D g) a, e\rangle
$$

and

$$
\langle L I a, I e\rangle=\langle I a, L I e\rangle=\left\langle I a,-\mu_{I}^{*}(D g)\right\rangle=-\left\langle I^{2} a,(D g)\right\rangle=\langle a, D g\rangle
$$

Similarly,

$$
\langle L J a, J e\rangle=\langle L K a, K e\rangle=\langle a, D g\rangle .
$$

The sum is zero. Hence we have that with respect to the decomposition $\mathcal{H} \oplus \mathcal{H}^{\perp}$

$$
|x|^{2}\left(D^{2} u\right)_{\mathbf{H}}=\left(\begin{array}{cc}
\operatorname{Hess}_{\mathbf{H}}^{\mathbf{P}} g-2 g I & 0 \\
0 & 0
\end{array}\right) .
$$

The $(1,1)$-term is the pull-back of the quaternionic hermitian symmetric part of the hessian on quaternionic projective space. This completes the proof. 


\section{Appendix A. Further Discussion of Examples.}

In this appendix we examine specific subequations of Riesz characteristic $p, 1 \leq p<\infty$ in more detail. We consider two types: cone subequations and convex cone subequations, and in both cases the subequations will always be ST-invariant . It may be of some surprise that in each of these two categories there is a unique largest and smallest subequation.

\section{The Largest / Smallest Characteristic $p$ Subequation.}

We first consider the category of cone subequations. For $A \in \operatorname{Sym}^{2}\left(\mathbf{R}^{n}\right)$ let $\lambda_{1}(A) \leq$ $\cdots \leq \lambda_{n}(A)$ denote the ordered eigenvalues of $A$, and set $\lambda_{\min }(A) \equiv \lambda_{1}(A)$ and $\lambda_{\max }(A) \equiv$ $\lambda_{n}(A)$. We then define

$$
\begin{aligned}
F_{p}^{\min / \max } & \equiv\left\{A: \lambda_{\min }(A)+(p-1) \lambda_{\max }(A) \geq 0\right\} \\
F_{p}^{\min / 2} & \equiv\left\{A: \lambda_{\min }(A)+(p-1) \lambda_{2}(A) \geq 0\right\}
\end{aligned}
$$

It is clear from Definition 3.4 that both of these subequations has Riesz characteristic $p$. These are the largest and smallest cone subequations with this property.

Lemma A.1. Let $F$ be an ST-invariant cone subequation of Riesz characteristic $p$. Then

$$
F_{p}^{\min / 2} \subset F \subset F_{p}^{\min / \max }
$$

Note. One computes that the dual of this largest subequation $F_{p}^{\min / \max }$ is $F_{q}^{\min / \max }$ where $(p-1)(q-1)=1$. Compare this with (3.15) in Part I which says that $\left(p_{F}-1\right)\left(q_{F}-1\right) \geq 1$ for any subequation $F$. Also see Example 10.14 in Part I.

Proof of Lemma A.1. Each $A \in \operatorname{Sym}^{2}\left(\mathbf{R}^{n}\right)$ can be written as a sum $A=\lambda_{1} P_{e_{1}}+$ $\cdots+\lambda_{n} P_{e_{n}}$ using the ordered eigenvalues of $A$. Set $B_{0} \equiv \lambda_{1} P_{e_{1}}+\lambda_{2} P_{e_{1}^{\perp}}$, and $B_{1} \equiv$ $\lambda_{1} P_{e_{1}}+\lambda_{n} P_{e_{1}^{\perp}}$, and note that $B_{0} \leq A \leq B_{1}$.

If $A \in F_{p}^{\min / 2}$, then $\lambda_{1}+(p-1) \lambda_{2} \geq 0$. Thus, $B_{0} \in F_{p}^{\min / 2}$. Since $F_{p}^{\min / 2}$ and $F$ have the same increasing radial profile $E^{\uparrow}$ given by (3.1) in Part I (and $\lambda_{2} \geq 0$ ), we conclude that $B_{0} \in F$. However, $B_{0} \leq A$ proving that $A \in F$.

For the other inclusion, pick $A \in F$. Since $F \subset \widetilde{\mathcal{P}}$, we have $\lambda_{\max } \geq 0$. Now $A \leq B_{1}$ implies $B_{1} \in F$. Again $F$ and $F_{p}^{\min / \max }$ have the same increasing radial profile $E^{\uparrow}$ given by (3.1) in Part I. Therefore, $B_{1} \in F_{p}^{\min / \max }$. This implies by definition that $A \in F_{p}^{\min / \max }$

The largest and smallest characteristic $p$ subequations in the convex cone case are different in dimensions $\geq 3$ (see Section 4 in Part I for the definitions of $\mathcal{P}_{p}$ and $\mathcal{P}(\delta)$ ).

Lemma A.2. Let $F$ be an $O(n)$-invariant convex cone subequation of Riesz characteristic $p$. Then $1 \leq p \leq n$ and

$$
\mathcal{P}_{p} \subset F \subset \mathcal{P}(\delta) \quad \text { where } \delta=\frac{(p-1) n}{n-p}
$$

and so the Riesz characteristic of $\mathcal{P}(\delta)$ is $p$. 
Proof. The first inclusion follows from the fact that $-(p-1) P_{e}+P_{e^{\perp}}$ generate the extreme rays in $\mathcal{P}_{p}$. This is proved in $\left[\mathrm{HL}_{7}\right.$, Thm. 5.1c]. The second inclusion is Proposition 10.9 in Part I.

\section{O(n)-Invariant Subequations.}

Such a subequation $F$ determines a subset $E \subset \mathbf{R}^{n}$ consisting of the $n$-tuples $\left(\lambda_{1}(A), \ldots, \lambda_{n}(A)\right)$ of eigenvalues of $A$. Consider $\lambda(A)=\left(\lambda_{1}(A), \ldots, \lambda_{n}(A)\right)$ as a multi-valued map $\lambda$ : $\operatorname{Sym}^{2}\left(\mathbf{R}^{n}\right) \rightarrow \mathbf{R}^{n}$. Then we define $E \equiv \lambda(F)$. The set $E$ is closed and symmetric (invariant under the permutation of coordinates in $\mathbf{R}^{n}$ ). In addition,

$$
E \text { is } \mathbf{R}_{+}^{n} \text { (positive orphant) monotone, i.e., } E+\mathbf{R}_{+}^{n}=E \text {, }
$$

since the ordered eigenvalues are $\mathcal{P}$-monotone.

Definition A.3. A closed symmetric subset $E \subset \mathbf{R}^{n}\left(\right.$ with $\left.\emptyset \neq E \neq \mathbf{R}^{n}\right)$ will be called a universal eigenvalue subequation if $E$ is $\mathbf{R}_{+}^{n}$-monotone.

Note that this is an abuse of language since $E$ itself is not a subequation. The "universal" nature of $E$ will be described later. However, such a set $E$ determines the $\mathrm{O}(n)$-invariant subequation

$$
F=\lambda^{-1}(E) .
$$

Note that

$$
\begin{array}{cl}
F \text { is a cone } & \Longleftrightarrow \quad E \text { is a cone, and } \\
F \text { is convex } & \Longleftrightarrow \quad E \text { is convex. }
\end{array}
$$

Of course, $\mathcal{P}$ and $\mathbf{R}_{+}^{n}$ correspond, i.e., $\mathcal{P}=\lambda^{-1}\left(\mathbf{R}_{+}^{n}\right)$. The Riesz characteristic of $F$ is easily computed from its eigenvalue profile $E$.

The increasing Riesz characteristic of $F$ equals $\sup \{p:(-(p-1), 1, \ldots, 1) \in E\}$.

The decreasing Riesz characteristic of $F$ equals $\sup \{q:(-1, \ldots,-1,(q-1)) \in E\}$.

It is also worth noting that if $E$ and $F$ correspond, the $\widetilde{E}$ and $\widetilde{F}$ correspond.

\section{The Complex and Quaternionic Analogues of an O(n)-Invariant Subequation.}

As described in Example 4.7 each $\mathrm{O}(n)$-invariant subequation $F$ on $\mathbf{R}^{n}$ canonically determines a $\mathrm{U}(n)$-invariant subequation $F^{\mathbf{C}}$ on $\mathbf{C}^{n}$ and an $\mathrm{Sp}(\mathrm{n})$-invariant subequation $F^{\mathbf{H}}$ on $\mathbf{H}^{n}$. In both cases the subequation is given by requiring that the $n$ eigenvalues of the hermitian symmetric part of the matrix lie in $E_{F}$. That is, if $E$ is defined by: $A \in F \Longleftrightarrow \lambda(A) \in E$, then

$$
A \in F^{\mathbf{C}} \Longleftrightarrow \lambda_{k}^{\mathbf{C}}(A) \in E \quad \text { and } \quad A \in F^{\mathbf{H}} \Longleftrightarrow \lambda_{k}^{\mathbf{H}}(A) \in E
$$

where $\lambda_{k}^{\mathbf{C}}(A)=\lambda_{k}\left(A_{\mathbf{C}}\right)$ and $\lambda_{k}^{\mathbf{H}}(A)=\lambda_{k}\left(A_{\mathbf{H}}\right)$ 
The associated Riesz characteristics are given in Lemma 4.9.

This classifies all the $\mathrm{U}(n)$-invariant subequations $F$ with the property that:

$$
F=\pi_{\mathbf{C}}^{-1}\left(F^{\mathbf{C}}\right) \quad \text { where } \pi_{\mathbf{C}}(A)=A_{\mathbf{C}}
$$

and $F^{\mathbf{C}}$ is a subset of the hermitian symmetric matrices. Similarly, it defines all the $\operatorname{Sp}(n)$-invariant subequations $F$ with the property that:

$$
F=\pi_{\mathbf{H}}^{-1}\left(F^{\mathbf{H}}\right) \quad \text { where } \pi_{\mathbf{H}}(A)=A_{\mathbf{H}}
$$

and $F^{\mathbf{H}}$ is a subset of the quaternionic hermitian symmetric matrices.

The largest / smallest results (Lemmas A.1 and A.2 in the $\mathbf{R}$ case) have counterparts in the $\mathbf{C}$ and $\mathbf{H}$ cases. The precise statements and their proofs are left to the reader.

Example A.4. (Lagrangian Subharmonic). A notable new example of a U(n)invariant subequation not satisfying (A.8) comes from Lagrangian geometry, namely, the geometrically defined subequation $F(\mathrm{LAG})$ where LAG $\subset G^{\mathbf{R}}\left(n, \mathbf{C}^{n}\right)$ is the set of Lagrangian $n$-planes in $\mathbf{C}^{n}$. The eigenvalues of the skew-hermitian part $A_{\mathbf{C}}^{\text {skew }}$ come in pairs $\lambda_{1},-\lambda_{1}, \lambda_{2},-\lambda_{2}, \ldots, \lambda_{n},-\lambda_{n}$. The subequation $F(\mathrm{LAG})$ is determined by a constraint on these eigenvalues together with the real trace $t=\operatorname{tr}(A)$, namely

$$
\frac{t}{2} \pm \lambda_{1} \pm \lambda_{2} \pm \cdots \pm \lambda_{n} \geq 0
$$

for all $2^{n}$ choices of \pm . There is a polynomial operator $M$ on $\operatorname{Sym}_{\mathbf{R}}^{2}\left(\mathbf{C}^{n}\right)$ analogous to the determinants $\operatorname{det}_{\mathbf{R}} A, \operatorname{det}_{\mathbf{C}} A$ and $\operatorname{det}_{\mathbf{H}} A$ (defined as the product of the eigenvalues in all cases), namely

$$
M_{\mathrm{LAG}}(A)=\prod_{2^{n} \text { times }}\left(\frac{t}{2} \pm \lambda_{1} \pm \lambda_{2} \pm \cdots \pm \lambda_{n}\right)
$$

(see $\left[\mathrm{HL}_{3}\right.$, page 433]). Of course since LAG $\subset G\left(n, \mathbf{R}^{2 n}\right)$ and $F$ is geometrically defined by LAG, $F$ has Riesz characteristic $n$.

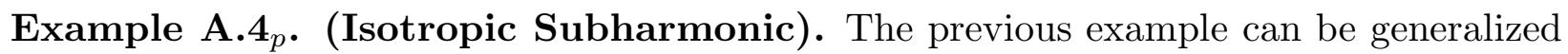
as follows. For each integer $p, 1 \leq p \leq n$ we consider the set

$$
\mathrm{ISO}_{p}=\left\{W \in G^{\mathbf{R}}\left(p, \mathbf{C}^{n}\right): W \text { is an isotropic } p \text { plane }\right\} .
$$

Recall that a real $p$-plane $W$ in $\mathbf{C}^{n}=\mathbf{R}^{2 n}$ is isotropic if

$$
v \perp J w \quad \forall v, w \in W
$$

i.e., the Kähler form $\omega$ satisfies $\left.\omega\right|_{W}=0$. Note that $\mathrm{ISO}_{n}=\mathrm{LAG}$ and $\mathrm{ISO}_{1}=\mathcal{P}$. For all $p>1$, the set $\mathrm{ISO}_{p}$ has the transitivity property, and so Theorem 3.2 applies. 
Associated to this set is the subequation

$$
\begin{aligned}
F\left(\mathrm{ISO}_{p}\right) & =\left\{A \in \operatorname{Sym}^{2}\left(\mathbf{R}^{2 n}\right): \forall W \in \mathrm{ISO}_{p}, \operatorname{tr}_{W} A \geq 0\right\} \\
& =\left\{A \in \operatorname{Sym}^{2}\left(\mathbf{R}^{2 n}\right): \frac{p}{n} t-\lambda_{n-p+1}-\cdots-\lambda_{n} \geq 0\right\}
\end{aligned}
$$

where $0 \leq \lambda_{1} \leq \cdots \leq \lambda_{n}$ are as in Example A.4. The dual subequation is

$$
\begin{aligned}
\widetilde{F}\left(\mathrm{ISO}_{p}\right) & =\left\{A \in \operatorname{Sym}^{2}\left(\mathbf{R}^{2 n}\right): \exists W \in \mathrm{ISO}_{p}, \operatorname{tr}_{W} A \geq 0\right\} \\
& =\left\{A \in \operatorname{Sym}^{2}\left(\mathbf{R}^{2 n}\right): \frac{p}{n} t+\lambda_{n-p+1}+\cdots+\lambda_{n} \geq 0\right\}
\end{aligned}
$$

Associated to this problem we have the polynomial

$$
\mathbf{M}_{\mathrm{ISO}_{p}}(A)=\prod_{|I|=p \text { and } \pm}\left(\frac{p}{n} t \pm \lambda_{i_{1}} \pm \cdots \pm \lambda_{i_{p}}\right)
$$

which is a factor of $\operatorname{det}\left(D_{A_{L A G}}\right)$ where $D_{A_{L A G}}: \Lambda^{p} \mathbf{R}^{2 n} \rightarrow \Lambda^{p} \mathbf{R}^{2 n}$ is the extension of $A_{L A G}$ as a derivation. As above we have that any $C^{2}$ function $u$ which is $\mathrm{ISO}_{p}$-harmonic satisfies the differential equation

$$
\mathbf{M}_{\mathrm{ISO}_{p}}(\operatorname{Hess} u)=0
$$

\section{Subequations Arising from Gårding Operators.}

Gårding's beautiful theory of hyperbolic polynomials provides a surprisingly rich collection of nonlinear operators. (This connection is mentioned in Krylov [Kr]). Moreover, associated with each such "Gårding operator" there are many actual subequations. Here we provide a brief overview. We first start with an operator and discuss how the many associated subequations are universally constructed. We then describe three of the basic ways of constructing new Gårding operators from a given one. These repeatable processes lead to a vast array of Gårding operators, starting with just one.

See for example $\left[\mathrm{HL}_{5}\right]$ for a self-contained development of Gårding's theory. His two fundamental results can be summarized by saying that:

The Garding cone $\Gamma$ is convex, and

The Garding eigenvalues are $\Gamma-$ monotone.

Definition A.5. A homogeneous real polynomial $M$ of degree $m$ on the space $\operatorname{Sym}^{2}\left(\mathbf{R}^{n}\right)$ of second derivatives, with $M(I)>0$, is a Gårding operator if:

(1) For each $A \in \operatorname{Sym}^{2}\left(\mathbf{R}^{n}\right)$ the polynomial $M(s I+A)$ has $m$ real $\operatorname{roots}(M$ is $I$ hyperbolic), and

(2) The Gårding cone $\Gamma$, defined as the connected component of $I$ in $\{M(A)>0\}$, satisfies positivity $\Gamma+\mathcal{P} \subset \Gamma$. 
The primary subequation associated with the Gårding operator $M$ is the closure of the Gårding cone $\Gamma$, which is a convex cone subequation. However, there are many others.

Definition A.6. The negatives of the roots of $M(s I+A)=0$ are called the $M$-eigenvalues of $A$, and are denoted by $\lambda^{M}(A) \equiv\left(\lambda_{1}^{M}(A), \ldots, \lambda_{m}^{M}(A)\right)$. They are well defined up to permutations. Note that $M(A)=\lambda_{1}^{M}(A) \cdots \lambda_{n}^{M}(A)$.

Definition A.7. The $\mathbf{k}^{\text {th }}$ branch of the equation $M(A)=0$ is defined to be the set

$$
\left\{\lambda_{k}^{M}(A) \geq 0\right\}
$$

where $\lambda_{1}^{M}(A) \leq \cdots \leq \lambda_{m}^{M}(A)$ are the ordered eigenvalues of $A$.

An important part of the theory shows that the ordered eigenvalues are strictly $\Gamma$ monotone. Since $\mathcal{P} \subset \bar{\Gamma}$ by (A.13), we have that each of the $m$ branches of $\{M(A)=0\}$ is a subequation.

Note that $\bar{\Gamma}=\left\{\lambda_{\min }^{M}(A) \geq 0\right\}$ is the primary branch, and its dual subequation is $\left\{\lambda_{\max }^{M}(A) \geq 0\right\}$, which is the largest branch.

The branches $\left\{\lambda_{k}^{M}(A) \geq 0\right\}, k=1, \ldots, m$, are the subequations most intimately associated with the Garding operator $M$ in that if a $C^{2}$-function $u$ is harmonic for one of these subequations, then

$$
M\left(D_{x}^{2} u\right)=0 .
$$

However, there are many others, all constructed exactly as in the $\mathrm{O}(\mathrm{n})$-invariant case.

Now we make full use of the concept (Def. A.3) of a universal eigenvalue subequation.

Proposition A.8. Given a universal eigenvalue subequation $E \subset \mathbf{R}^{m}$, each Gårding operator of degree $m$ on $\operatorname{Sym}^{2}\left(\mathbf{R}^{n}\right)$ determines a subequation on $\mathbf{R}^{n}$, namely

$$
F_{E} \equiv\left\{A: \lambda^{M}(A) \in E\right\} .
$$

This subequation is $\bar{\Gamma}$-monotone (not just $\mathcal{P}$-monotone). Moreover, $F_{E}$ is a cone if and only if $E$ is a cone., and $F_{E}$ is convex if and only if $E$ is convex.

Proof. This is straightforward except for the last assertion which is due to [BGLS].

For example, $E=\mathbf{R}_{+}^{m}$ is the universal "Monge-Ampère subequation" inducing the subequation $\bar{\Gamma}$ for each degree $m$ Gårding operator $M(A)=\lambda_{1}^{M}(A) \cdots \lambda_{m}^{M}(A)$.

We complete this discussion of Gårding operators by describing three of the basic methods of constructing new Gårding operators from a given Gårding operator $M$ of degree $m$. To be specific the reader may want to start with one of the basic operators $\operatorname{det}\left(A_{K}\right)$ for $K=\mathbf{R}, \mathbf{C}$ or $\mathbf{H}$.

I. The Derived or Elementary Symmetric Operator. With $k=1, \ldots, m$ we set

$$
\left.\sigma_{k}(A) \equiv \frac{1}{(n-k) !} \frac{d^{m-k}}{d t^{m-k}} M(A+t I)\right|_{t=0}=\sum_{i_{1}<\cdots i_{k}} \lambda_{i_{1}}^{M}(A) \cdots \lambda_{i_{k}}^{M}(A)
$$


II. The $p$-Convexity Operator. For each real number $p$ with $1 \leq p \leq m$ set

$$
\Sigma_{p}(A) \equiv \prod\left\{\lambda_{i_{1}}^{M}(A)+\cdots+\lambda_{i_{[p]}}^{M}(A)+(p-[p]) \lambda_{j}^{M}(A)\right\}
$$

where the product is taken over all increasing multi-indicies $I=\left(i_{1}, \ldots, i_{[p]}\right)$ and all $j \notin I$.

III. The $\delta$-Uniformly Elliptic Regularization Operator. With $0 \leq \delta \leq \infty$ (and renormalizing at $\delta=\infty$ ) set

$$
M^{\delta}(A) \equiv \prod_{j=1}^{m}\left\{\lambda_{j}^{M}(A)+\frac{\delta}{n}(\operatorname{tr} A)\right\}
$$

Remark A.9. The first process lowers the degree of the operator. The second process raises the degree of the operator, and the degree remains the same in the third process. Each of these construction can be applied repeatedly. In the third process nothing new is obtained from this iteration. However, iterating the first process produces a finite number of new Gårding operators, and iterating the second produces an infinite family of new ones. Moreover, one can apply any sequence of the three operations, thereby producing a huge collection of Gårding operators all dependent on the primary operator.

\section{Elliptic Regularization - Subequation Expansion/Contraction.}

For each $r>0$ consider the linear map

$$
\Phi_{r}(A) \equiv r A+(1-r)(\operatorname{tr} A) \frac{1}{n} I=r\left(A-(\operatorname{tr} A) \frac{1}{n} I\right)+(\operatorname{tr} A) \frac{1}{n} I .
$$

The restriction of $\Phi_{r}$ to each affine hyperplane $\{\operatorname{tr} A=\lambda\}$ is the $r$-homothety (multiplication by $r$ ) about the center $\frac{\lambda}{n} I$. This follows from the second equality. The inverse is

$$
\Phi_{\frac{1}{r}}=\frac{1}{r}\left(A+(r-1)(\operatorname{tr} A) \frac{1}{n} I\right) .
$$

Definition A.10. Suppose $\delta \equiv r-1 \geq 0$ and $F$ is a cone subequation. Then

$$
F(\delta)=\Phi_{r}(F)=\left\{A: r \Phi_{\frac{1}{r}}(A)=A+\delta(\operatorname{tr} A) \frac{1}{n} I \in F\right\}
$$

is called the $\mathbf{r}^{\text {th }}$ expansion of $F$.

Note that $F(\delta)$ is also a subequation for all $\delta>0$ since the homothety factor $r \geq 1$. Note also that if $F$ is a convex cone contained in $\operatorname{Int} \Delta=\{\operatorname{tr} A>0\}$, then $F(\delta)$ ranges from $F$ to $\Delta$ as $\delta$ ranges from 0 to $\infty$. Finally, note that $\partial F(\delta)=\Phi_{r}(\partial F)$.

Proposition A.11. Suppose $F$ is a cone subequation with (Riesz) characteristic $p=p_{F}$ and dual (Riesz) characteristic $q=q_{F}$. Then the $\delta$-uniformly elliptic cone subequation $F(\delta)=\Phi_{r}(F)(\delta \equiv r-1 \geq 0)$ has its two characteristics given by the same function

$$
\begin{aligned}
& p_{F(\delta)}=\frac{n(1+\delta) p}{n+\delta p}=p+\frac{\delta p(n-p)}{n+\delta p} \\
& q_{F(\delta)}=\frac{n(1+\delta) q}{n+\delta q}=q+\frac{\delta q(n-q)}{n+\delta q}
\end{aligned}
$$


These formulas hold when $p_{F}=\infty$ or when $q_{F}=\infty$, that is

$$
\begin{aligned}
& p_{F}=\infty \quad \Rightarrow \quad p_{F(\delta)}=\frac{n(1+\delta)}{\delta} \\
& q_{F}=\infty \quad \Rightarrow \quad q_{F(\delta)}=\frac{n(1+\delta)}{\delta}
\end{aligned}
$$

Proof. Note that $A \equiv P_{e^{\perp}}-(p-1) P_{e} \in \partial F \Longleftrightarrow \Phi_{r}(A) \in \partial \Phi_{r}(F)=\partial F(\delta)$ and

$\Phi_{r}(A)=(1+\delta) P_{e^{\perp}}-(1+\delta)(p-1) P_{e}-\frac{\delta(n-p)}{n} I=\frac{n+\delta p}{n}\left[P_{e^{\perp}}-\left(\frac{n(1+\delta) p}{n+\delta p}-1\right) P_{e}\right]$

Finally, since $-A \in \partial F \Longleftrightarrow-\Phi_{r}(A) \in \partial \Phi_{r}(F)=\partial F(\delta)$, the formula for $q_{F(\delta)}$ as a function of $q_{F}$ is the same as the formula for $p_{F(\delta)}$ as a function of $p_{F}$.

Proposition A.12. If $F$ is $M$-monotone, then $F(\delta)$ is $M(\delta)$-monotone.

Proof. Straightforward.

Example A.13. As $\delta$ ranges from 0 to $\infty, \mathcal{P}(\delta)$ increases from $\mathcal{P}$ to $\Delta$. Each $\mathcal{P}(\delta)$ is a convex cone ; and with $\delta>0$ small, these subequations form a "fundamental system" of conical neighborhoods of $\mathcal{P}$. Consequently, they provide one of the nicer definitions of uniform ellipticity. Namely, a subequation $F$ is $\delta$-uniformly elliptic if

$$
F+\mathcal{P}(\delta) \subset F
$$

Since $F(\delta)+\mathcal{P}(\delta) \subset F(\delta)$, each $F(\delta)$ is automatically $\delta$-uniformly elliptic. For this reason, $F(\delta)$ is also called the $\delta$-elliptic regularization of $F$ (cf. $[\mathrm{Kr}]$ ). 


\section{REFERENCES}

$\left[\mathrm{A}_{1}\right]$ S. Alesker, Non-commutative linear algebra and plurisubharmonic functions of quaternionic variables, Bull. Sci. Math., 127 (2003), 1-35. also ArXiv:math.CV/0104209.

$\left[\mathrm{A}_{2}\right] \longrightarrow$, Quaternionic Monge-Ampère equations, J. Geom. Anal., 13 (2003), 205-238. ArXiv:math.CV/0208805.

[AV] S. Alesker and M. Verbitsky, Plurisubharmonic functions on hypercomplex manifolds and HKT-geometry, J. Geom. Anal. 16 (2006), no. 3, 375399.

[BGLS] H. Bauschke, O. Güler, A. Lewis and H. Sendov, Hyperbolic polynomials and convex analysis, Canad. J. Math. 53 no. 3, (2001), 470-488.

[HL $\mathrm{HL}_{1}$ F. R. Harvey and H. B. Lawson, Jr, Calibrated geometries, Acta Mathematica 148 (1982), 47-157.

$\left[\mathrm{HL}_{2}\right] \longrightarrow$, An introduction to potential theory in calibrated geometry, Amer. J. Math. 131 no. 4 (2009), 893-944. ArXiv:math.0710.3920.

$\left[\mathrm{HL}_{3}\right] \longrightarrow$, Dirichlet duality and the non-linear Dirichlet problem, Comm. on Pure and Applied Math. 62 (2009), 396-443. ArXiv:math.0710.3991

$\left[\mathrm{HL}_{4}\right] \longrightarrow$, Dirichlet duality and the nonlinear Dirichlet problem on Riemannian manifolds, J. Diff. Geom. 88 (2011), 395-482. ArXiv:0912.5220.

[HL $\left.\mathrm{HL}_{5}\right]$ - Gårding's theory of hyperbolic polynomials, Comm. Pure Appl. Math. 66 (2013), no. 7, 11021128.

$\left[\mathrm{HL}_{6}\right] \longrightarrow$, The restriction theorem for fully nonlinear subequations, Ann. Inst. Fourier (to appear). ArXiv:1101.4850.

$\left[\mathrm{HL}_{7}\right] \longrightarrow$, p-convexity, p-plurisubharmonicity and the Levi problem , Indiana Univ. Math. J. 62 No. 1 (2014), 149-170. ArXiv:1111.3895.

$\left[\mathrm{HL}_{8}\right] \longrightarrow$, Existence, uniqueness and removable singularities for nonlinear partial differential equations in geometry, pp. 102-156 in "Surveys in Differential Geometry 2013", vol. 18, H.-D. Cao and S.-T. Yau eds., International Press, Somerville, MA, 2013. ArXiv:1303.1117.

$\left[\mathrm{HL}_{9}\right] \longrightarrow$ - Removable singularities for nonlinear subequations, ArXiv:1303.0437.

$\left[\mathrm{HL}_{10}\right] \longrightarrow$, Tangents to subsolutions - existence and uniqueness, I, (to appear).

[K] C. Kiselman, Tangents of plurisubharmonic functions, International Symposium in Memory of Hua Loo Keng, Vol. II (Beijing, 1988), 157167, Springer, Berlin, 1991.

$[\mathrm{Kr}]$ N. V. Krylov, On the general notion of fully nonlinear second-order elliptic equations, Trans. Amer. Math. Soc. (3) 347 (1979), 30-34.

[R] R, T. Rockafellar, Convex analysis. Princeton Mathematical Series, No. 28, Princeton University Press, Princeton, N.J., 1970. 